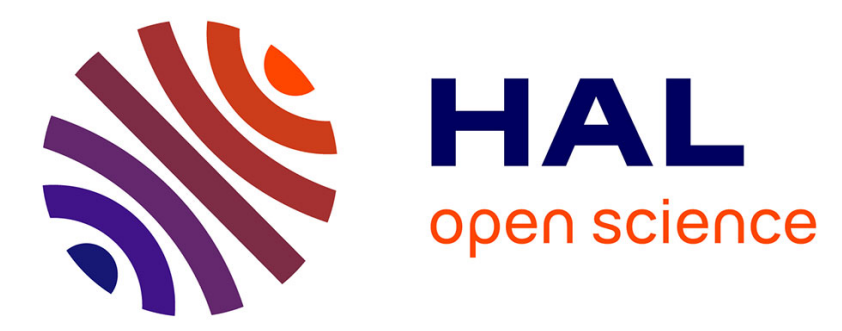

\title{
Biocompatible Glyconanoparticles by Grafting of Sophorolipid Monolayers on Monodisperse Iron Oxide Nanoparticles
}

Andrea Lassenberger, Andrea Scheberl, Krishna Chaithanya Batchu, Viviana Cristiglio, Isabelle Grillo, Daniel Hermida Merino, Erik Reimhult, Niki Baccile

\section{To cite this version:}

Andrea Lassenberger, Andrea Scheberl, Krishna Chaithanya Batchu, Viviana Cristiglio, Isabelle Grillo, et al.. Biocompatible Glyconanoparticles by Grafting of Sophorolipid Monolayers on Monodisperse Iron Oxide Nanoparticles. ACS Applied Bio Materials, 2019, 2 (7), pp.3095-3107. 10.1021/acsabm.9b00427 . hal-02164319

\section{HAL Id: hal-02164319 https://hal.sorbonne-universite.fr/hal-02164319}

Submitted on 25 Jun 2019

HAL is a multi-disciplinary open access archive for the deposit and dissemination of scientific research documents, whether they are published or not. The documents may come from teaching and research institutions in France or abroad, or from public or private research centers.
L'archive ouverte pluridisciplinaire HAL, est destinée au dépôt et à la diffusion de documents scientifiques de niveau recherche, publiés ou non, émanant des établissements d'enseignement et de recherche français ou étrangers, des laboratoires publics ou privés. 
This document is confidential and is proprietary to the American Chemical Society and its authors. Do not copy or disclose without written permission. If you have received this item in error, notify the sender and delete all copies.

\section{Biocompatible Glyconanoparticles by Grafting of Sophorolipid Monolayers on Monodisperse Iron Oxide Nanoparticles}

\begin{tabular}{|r|l|}
\hline Journal: & ACS Applied Bio Materials \\
\hline Manuscript ID & mt-2019-004275.R2 \\
\hline Manuscript Type: & Article \\
\hline Duthor: & 14-Jun-2019 \\
\hline Complete List of Authors: & $\begin{array}{l}\text { Lassenberger, Andrea; Institut Laue-Langevin, } \\
\text { Scheberl, Andrea; University of Natural Resources and Life Sciences } \\
\text { Batchu, Krishna; Institut Laue-Langevin } \\
\text { Cristiglio, Viviana; Institut Laue-Langevin, } \\
\text { Grillo, Isabelle; Institut Laue-Langevin, Large Scale Structures Group } \\
\text { Hermida-Merino, Daniel; Netherlands Organization for Scientific } \\
\text { Research, Chemistry } \\
\text { Reimhult, Erik; University of Natural Resources and Life Sciences Vienna, } \\
\text { Department of Nanobiotechnology } \\
\text { Baccile, Niki; CNRS-UPMC, LCMCP UPMC }\end{array}$ \\
\hline
\end{tabular}

\section{SCHOLARONE Manuscripts}




\title{
Biocompatible Glyconanoparticles by Grafting of
}

\section{Sophorolipid Monolayers on Monodisperse Iron}

\section{Oxide Nanoparticles}

Andrea Lassenberger, ${ }^{a, b}$ Andrea Scheberl, ${ }^{a}$, Krishna Chaithanya Batchu, ${ }^{b}$ Viviana Cristiglio, ${ }^{b}$ Isabelle Grillo, ${ }^{b}$ Daniel Hermida Merino, ${ }^{c}$ Erik Reimhult, ${ }^{a,}$ Niki Baccile ${ }^{d, *}$

aUniversity of Natural Resources and Life Sciences Vienna, Institute for Biologically

inspired materials, Department of Nanobiotechnology, Muthgasse 11/II, 1190 Vienna,

\author{
Austria \\ *erik.reimhult@boku.ac.at \\ bInstitut Laue-Langevin, 71 Avenue des Martyrs, 38042 Grenoble Cedex 9, France \\ 'ESRF - The European Synchrotron, 71 Avenue des Martyrs, 38042 Grenoble Cedex 9, \\ France
}




\author{
dSorbonne Université, Centre National de la Recherche Scientifique, Laboratoire de \\ Chimie de la Matière Condensée de Paris, LCMCP, F-75005 Paris, France \\ *niki.baccile@upmc.fr
}

KEYWORDS: sophorolipid, glyconanoparticle, nanoparticle surface functionalization, small angle neutron scattering, small angle X-ray scattering, lipid shell, iron oxide nanoparticle, core-shell nanoparticle, colloidal stability

\begin{abstract}
This work presents synthesis and characterization of sophorolipid-coated monodisperse iron oxide nanoparticles. Sophorolipids are biological glycosylated amphiphiles produced by the yeast $S$. bombicola. In their open acidic form, sophorolipids have been used as surface stabilizing agent for metal and metal oxide nanoparticles but with poor control over size and structural properties. In this work, the $\mathrm{COOH}$ function of sophorolipids (SL) was modified with nitrodopamine (NDA), a catechol known for its high affinity to iron ions. The resulting new form of sophorolipid-nitrodopamide (SL-NDA) was
\end{abstract}


used as surface ligand for monodisperse iron oxide nanoparticles. We show, by a combination of thermogravimetric analysis and small angle X-ray and neutron scattering, that iron oxide nanoparticles (IONP) are stabilized by a single, high-density SL-NDA layer, which results in excellent colloidal stability under biologically relevant conditions such as high protein and salt concentration. The IONP grafted with SL-NDA showed negligible uptake and no cytotoxicity tested on two representative cell lines. Thus, they reveal the potential of sophorolipids as stable and non-toxic surface coatings for IONP-based biomedical and biotechnological applications.

Introduction

Superparamagnetic iron oxide nanoparticles are used in applications such as drug delivery, MRI or hyperthermia, for which iron oxide cores smaller than $20 \mathrm{~nm}$ in diameter are coated with weakly adsorbed polymers (often dextran ${ }^{1-3}$ ) or lipids. ${ }^{4}$ However, physisorbed shells lead to low dispersant densities on the particle surface and low colloidal stability over time. Controlled grafting of chemically linked dispersants yields 
denser and more stable shells; therefore, such approaches have received increasing attention. ${ }^{5-6}$ With improvements in the synthesis, monodisperse (SD $<5 \%$ ) iron oxide nanoparticles have been developed, ${ }^{7}$ but their hydrophobic (mostly oleic acid) shell of weakly adhering ligands is not compatible with biomedical applications. Consequently, the original ligands must be replaced by chemisorbed ligands that provide a water-soluble and sterically stabilizing shell, crucial for colloidal stability in biological fluids. Ligand exchange protocols for highly hydrated and chemically grafted ligands, mainly based on poly(ethylene glycol) (PEG), have therefore been developed. ${ }^{8}$ PEG provides excellent colloidal stability and long circulation times of the particles in the body due to limited opsonization (protein binding and elimination of the particles by the reticuloendothelial system), ${ }^{9}$ but PEG does not provide targeting specificity and has increasingly been claimed to be subject to antibody production. ${ }^{10}$ Coating of nanoparticles with biological or biomimetic ligands could address both these drawbacks; to this end, other surface ligands are tested, including carbohydrates.

Carbohydrate-grafted nanoparticles are generally referred to as glyconanoparticles ${ }^{11-12}$ and they are constituted by a nanoparticle core (e.g. gold or iron oxide) and a shell 
composed of a glycoconjugate, a term which refers to a molecule composed of a carbohydrate on one side and a binding group on the other. Since specific carbohydrates are known to selectively bind proteins, ${ }^{13-15}$ glyconanoparticles generally contain mannose, galactose, fucose or sialic acid, which are well-known saccharides targeting carbohydrate receptors on tumor cells. ${ }^{14,} 16$ The binding group must be selected with the type of core particle surface in mind. Typically, thiols are used to graft to gold nanoparticles while silanes are used to graft to oxide nanoparticles. ${ }^{12,} 16-17$ The main drawback of these fully synthetic systems is the complex synthesis of the glycoconjugate ligands, mainly due to the classically tedious sugar chemistry, and for this reason alternative routes to prepare glyconanoparticles are worth exploring.

In the past fifteen years, a number of research groups has shown the potential of using natural capping agents for metallic nanoparticles. ${ }^{18-19}$ However, the field is still in its infancy due to the poor control over both particle size distribution and composition of the shell. A promising group of novel capping agents are glycoconjugates obtained through microbial synthesis. This class of molecules, in particular sophorolipids (SL), is the most studied in the field of nanoparticle stabilization. SL are bolaform, non-toxic, bio-based 
glycolipids produced by the yeast Starmerella bombicola and characterized by a bulky sophorose headgroup and a free carboxyl group. ${ }^{20} \mathrm{SL}$ are known to have natural targeting abilities, anti-cancer ${ }^{21}$ and anti-bacterial ${ }^{22}$ properties. SL were previously used to stabilize metal nanoparticles $(\mathrm{Ag}, \mathrm{Co})^{23-25}$ and iron oxide nanoparticles (IONP). ${ }^{26}$ The interaction between $S L$ and the nanoparticles was attributed to the complexing of the accessible COO- group to the surface; however, low grafting densities, poor control over polydispersity, shell composition and even nanoparticle structure show that further enhancement of SL-coated iron oxide nanoparticle synthesis and structure are required for applications. Here, we propose a new approach based on the use of size-controlled monodisperse IONP and SL having the $\mathrm{COOH}$ group modified with nitrodopamine (NDA) via an amide bond. NDA is an anchor molecule that has been used to bind dispersants strongly to IONP. ${ }^{27-28}$ We demonstrate grafting of a monolayer SL shell by a combination of SANS and synchrotron SAXS. Furthermore, we show the resulting excellent colloidal stability of the SL-IONP in physiological buffers and protein concentrations, paired with absent cytotoxicity. This work opens opportunities for the application of glycosylated IONP obtained from natural glycoconjugates, combining strong colloidal stability and 
water solubility with the imaging, tracking and therapeutic abilities contributed by the

IONP core.

\section{Experimental Section}

Materials. All Chemicals were purchased from Sigma-Aldrich and all solvents from

Roth. Chemicals were used as received without further purification (see supporting information for details). Sophorolipids (Sopholiance, batch number: 11103A, dry content: $60 \pm 6 \%)$ are purchased from Soliance, France.

Synthesis of sophorolipids (SL). Acidic sophorolipids have been prepared from a commercial batch of a sophorolipid mixture using alkaline hydrolysis to convert the crude lactonic/acidic mixture into a fully acidic sophorolipid compound. Extraction and purification have been performed using the method $\mathrm{N}^{\circ} 2$ described earlier, ${ }^{29}$ to which one should refer for a typical ${ }^{1} \mathrm{H}$ solution NMR fingerprint. The compound used here is majorly (> 80\%) composed of the acidic subterminal C18:1-cis form that forms micelles. ${ }^{30}$

Synthesis of nitrodopamide-SL (SL-NDA) ligands. 6-Nitrodopamine-hemisulfate (NDA$\mathrm{HSO}_{4}$ ) was synthesized according to literature with slight modifications. ${ }^{31}$ SL-NDA was 
synthesized by (1-cyano-2-ethoxy-2-oxoethylidenaminooxy)dimethylamino-morpholinocarbenium hexafluorophosphate (COMU) mediated peptide-coupling reactions. ${ }^{32-33}$ Briefly, $100 \mathrm{mg}(0.16 \mathrm{mmol})$ of SL were pre-activated with $1.5 \mathrm{eq}$ COMU and 1 eq $\mathrm{N}-$ methylmorpholine in $7 \mathrm{~mL}$ dimethylformamide (DMF) at $\mathrm{RT}$ for 10 minutes. The mixture was cooled to $0^{\circ} \mathrm{C}$ and a mix of 1 eq NDA and 1 eq $N$-methylmorpholine in $5 \mathrm{~mL}$ DMF was slowly dropped to the activated acid. The mixture reacted for $1 \mathrm{~h}$ at $0{ }^{\circ} \mathrm{C}$ and subsequently $3 \mathrm{~h}$ at RT. The solvent was evaporated and $4 \mathrm{~mL}$ of Milli-Q were added to the crude product. The product was transferred into butanol $(\mathrm{BuOH})$ and extracted thrice with Milli$\mathrm{Q}$, once with $1.5 \mathrm{M} \mathrm{HCl}$ and finally washed with pure Milli-Q. BuOH was evaporated and the product was vacuum-dried. The coupling of SL to NDA was confirmed by FTIR and by solution ${ }^{1} \mathrm{H}$ NMR (see Figure $\mathrm{S} 1$ for details), showing the coexisting 1:1 ratio between SL and NDA together with the downfield shift, from $3.05 \mathrm{ppm}$ to $3.47 \mathrm{ppm}$, attributed to the $\alpha-\mathrm{CH}_{2}$ in NDA and respectively in an amine and amide environment. ${ }^{32,} 34$ One can also observe the parallel upfield shift, from $2.30 \mathrm{ppm}$ to $2.18 \mathrm{ppm}$ of $\alpha-\mathrm{CH}_{2}$ in SL for respectively an amine and an amide environment. NMR excludes the presence of unreacted free NDA, but we must acknowledge the presence of residual COMU and 
unreacted SL (between 10 and $20 \mathrm{~mol} \%$ with respect to SL-NDA), which due to similar physico-chemical properties were not removed by washing the sample. However, COMU and unreacted SL do not have the affinity to the iron oxide nanoparticle surface to displace oleic acid or interfere with the ligand displacement by SL-NDA.

Synthesis of iron oxide nanoparticles (IONP). Iron oxide NP (3-10 nm in diameter) coated with oleic acid (OA) were synthesized by thermal decomposition of iron pentacarbonyl according to a heat-up procedure originally proposed by Hyeon et al. ${ }^{35}$ that was slightly modified as described in ${ }^{8}$. Details for the molar ratios of reactants can be found in the supporting information (Table S1). Larger, $14.1 \mathrm{~nm}$ IONP were synthesized via a modified two-step method introduced first by Park et al. ${ }^{36}$ and previously by us. ${ }^{9}$ IONP cores with diameters of $3.1,4.6$ and $14.1 \mathrm{~nm}$ were used for functionalization with sophorolipids and further experiments.

Ligand exchange and purification. SL-IONP were synthesized according to a slightly modified method published earlier. ${ }^{8}$ For $4.6 \mathrm{~nm}$ cores, $0.9 \mathrm{~g}$ of as-synthesized IONP coated with oleic acid were dispersed in $20 \mathrm{~mL}$ DMF. $0.183 \mathrm{~g}$ of SL-NDA were added and the mixture was sonicated for $28 \mathrm{~h}$ at slightly elevated temperature. Amounts of ligand 
were adjusted for the smaller $3.1 \mathrm{~nm}$ IONP to account for the difference in surface area and the same surface area to ligand ratio was used. However, for the $14.1 \mathrm{~nm}$ cores we used a much larger excess of ligand (10x with respect to surface area), supposing that a larger excess of ligand will aid in achieving a high surface coverage. After ligand exchange, the SL-IONP were washed three times with n-hexane to remove released OA. In a final step, the solvent was evaporated and the IONP were lyophilized. The lyophilized SL-IONP were redispersed in Milli-Q and dialysed in a 12-14 kDa MWCO dialysis bag against $5 \mathrm{~L}$ Milli-Q to remove excess SL-NDA ligands. Alternatively, SL-IONP were purified by repeated (5x) membrane centrifugation in 50 kDa MWCO Amicon filter units. The purified SL-IONP were lyophilized and could be stored for more than two years as a dark-brown powder. The yield was $96 \%$ calculated with respect to the initial amount of IONP cores. SL-IONP were analyzed by TEM, TGA, ATR-FTIR and DLS.

The samples were named according to their core diameter measured by TEM: $3.1 \mathrm{~nm}$ SL-IONP (diameter $3.1 \mathrm{~nm}$ ), $4.6 \mathrm{~nm}$ SL-IONP (diameter $4.6 \mathrm{~nm}$ ) and $14.1 \mathrm{~nm}$ SL-IONP (diameter $14.1 \mathrm{~nm}$ )

Cell growth. U937 cells were grown under conditions reported in ${ }^{9}$. 
Toxicity test. Toxicity measurements were performed with the PrestoBlue® Cell Viability assay. Briefly, $2 \times 10^{4}$ cells were seeded on 96-well plates using $200 \mu \mathrm{l}$ medium per well and incubated for $16 \mathrm{~h}$ (each 6 repeats for sample and control). $5 \mu \mathrm{l}$ nanoparticle dispersion (1 $\mathrm{mg} \mathrm{Fe} / \mathrm{ml}$ ) were added and incubated for either 2 or $20 \mathrm{~h}$. As control $5 \mu \mathrm{l}$ Milli-Q water were added. After incubation, $20 \mu \mathrm{l}$ PrestoBlue ${ }^{\circledR}$ were added and plates were incubated for one hour. A Tecan infinite F200 plate reader with excitation filter $560 \pm 20$ $\mathrm{nm}$ and emission filter $595 \pm 35 \mathrm{~nm}$ was used to read the plates. The background was determined by measuring medium with presto blue at $10 \%(\mathrm{v} / \mathrm{v})$.

Viability was calculated as:

$$
\frac{x}{X_{C}} * 100 \%
$$

with $X$ the average value determined for cells incubated with SL-IONP and $X_{c}$ the average value measured for control cells.

SL-IONP uptake by cells and iron quantification. For uptake experiments, $2 \times 10^{6}$ cells (monocytes) and $1 \times 10^{6}$ cells (MCF-7) were seeded in a total volume of $5 \mathrm{ml}$ in 6-well plates and incubated for $12 \mathrm{~h} .50 \mu \mathrm{L}$ of a $1 \mathrm{mg} / \mathrm{mL}$ Fe dispersion of SL-IONP were added 
and the cells were incubated for 20 hours. The control well was incubated with $50 \mu \mathrm{L}$ Milli$Q$ water. After incubation, cells were centrifuged and consequently washed three times with PBS to remove IONP that were not taken up the cells. Iron quantification was done by a modified Ferrozine test as described previously. ${ }^{9}$ For each type of SL-IONP at least 5 wells were prepared in parallel and 3 independent repetitions were performed, resulting in a minimum of 9 data points for each type of SL-IONP.

Protein quantification. The Rotitm-NanoQuant protein detection assay was used for protein quantification according to the manufacturer's protocol. BSA dissolved in $50 \mathrm{mM}$ $\mathrm{NaOH}$ was used as a standard.

Transmission electron microscopy (TEM) and analysis. TEM images were recorded on a FEI Tecnai G2 20 transmission electron microscope at $160 \mathrm{kV}$ for low resolution and $200 \mathrm{kV}$ for high resolution imaging. Samples were drop-casted from toluene dispersions (IONP capped by oleic acid) or aqueous dispersions (SL-IONP) onto a 300-mesh carboncoated copper. The Pebbles ${ }^{37}$ software package was used to determine the size distributions from the micrographs. Approximately 900 IONP were used for size determination by Pebbles for each sample of nanoparticles. 


\section{Transmission Electron Microscopy experiments under cryogenic conditions (cryo-}

TEM). Cyro-TEM images were recorded on a FEI Tecnai 120 twin microscope at $120 \mathrm{kV}$ with a Gatan Orius CCD numeric camera and a Gatan Cryoholder (Gatan 626DH, Gatan).

DigitalMicrograph $^{\mathrm{TM}}$ software was used for image acquisition. A custom-built device was used for cryo-fixation. SL-IONP aqueous dispersions and SL-NDA solutions were deposited on glow-discharged holey carbon copper grids (Quantifoil R2/2, Germany). The grids were immediately plunge-freezed by dipping into liquid ethane at $-180{ }^{\circ} \mathrm{C}$ and transfered into liquid nitrogen. All grids were stored in liquid nitrogen.

Thermogravimetric Analysis (TGA). TGA data were measured using a Mettler-Toledo TGA/DSC 1 STAR System in a temperature range of $25-650^{\circ} \mathrm{C}$ with $10 \mathrm{~K} / \mathrm{min}$. A stream of $80 \mathrm{~mL} / \mathrm{s}$ of synthetic air was used to ensure complete combustion of the SL-NDA ligands since NDA is known to polymerize by pyrolysis under $\mathrm{N}_{2} .0 .5-1.5 \mathrm{mg}$ sample were measured, The total organic content (TOC) was calculated as the fraction of mass loss up to $650^{\circ} \mathrm{C}$.

Dynamic Light Scattering (DLS). DLS was measured on a Malvern Zetasizer Nano-ZS at $20{ }^{\circ} \mathrm{C}$. Instrument settings: $\mathrm{Fe}_{3} \mathrm{O}_{4}$, Milli-Q as dispersant $(0.89 \mathrm{mPa} \cdot \mathrm{s}, \mathrm{RI}=1.33), 3$ 
individual measurements were performed with 13 runs of $10 \mathrm{~s}$ each. All samples of SLIONP were measured at a concentration of $0.5 \mathrm{mg}_{\mathrm{Fe}} / \mathrm{mL}$. The nanoparticles were dispersed in water $(2 \mathrm{mg} / \mathrm{mL})$ and $10 \%$ fetal calf serum for experiments in which the temperature was varied from $20^{\circ} \mathrm{C}$ to $70^{\circ} \mathrm{C}$ and back with $5^{\circ} \mathrm{C}$ steps and an equilibration time of 2 minutes. At each temperature, 3 individual measurements with 13 runs of $10 \mathrm{~s}$ were recorded.

${ }^{1} \mathrm{H}$ Nuclear magnetic resonance (NMR) measurements. ${ }^{1} \mathrm{H}$ NMR data were acquired on a Bruker Avance III 300 spectrometer using a $5 \mathrm{~mm}{ }^{1} \mathrm{H}-\mathrm{X}$ BBFO probe, 16 transients with $1 \mathrm{~s}$ recycling delay. The value chemical shifts $(\delta)$ are reported in ppm at $298 \mathrm{~K}$ using tetramethylsilane (TMS) as reference. MeOD-d4 was used as solvent. COSY spectra were acquired using a standard pulse program form the Avance III library, with $1 \mathrm{~s}$ recycling delay, 8 transients per scan and 256 spectra acquired in the F1 dimension.

\author{
Attenuated total reflection - Fourier transform infra-red (ATR-FTIR) measurements. IR \\ spectra of the lyophilized samples were recorded on a Perkin Elmer 400 FTIR
}


spectrometer with diamond single-reflection ATR equipment and a resolution of $4 \mathrm{~cm}^{-1}$, averaging 32 scans.

Small Angle X-ray Scattering (SAXS). SAXS patterns were measured using a DectrisPilatus $1 \mathrm{M}$ detector with a resolution of $981 \times 1043$ pixels and a pixel size of $172 \times 172$ $\mu \mathrm{m}$ at the Dutch-Belgian Beamline (DUBBLE) station BM26B of the European Synchrotron Radiation Facility (ESRF) in Grenoble, France. The sample to SAXS detector distance was set to ca. $3100 \mathrm{~mm}$ using a wavelength of $1.033 \AA$. SAXS patterns were integrated azimuthally to obtain a typical $I(q)$ spectrum. A quartz capillary, used as sample holder, was filled with Milli-Q grade water, used as solvent, and then with the sample dispersion. To eliminate errors in the background subtraction process due to in homogeneities of the capillary walls, great care was taken to analyze both the water and sample solutions at the same position. Solvent contribution was subtracted during the data treatment. Data were corrected for the transmission of the direct beam and presented in absolute scale. The q-range calibration was made using a silver behenate standard sample $\left(d_{r e f}=58.38 \AA\right)$ 
Small Angle Neutron Scattering (SANS). SANS was performed at the Institut LaueLangevin (ILL, Grenoble, France) on D16 and D33 beamlines. The spectrometer configurations were adjusted as follows. D16 beamline $\left(3.5 \cdot 10^{-2} \AA^{-1}<q<0.5 \AA^{-1}\right)$ : neutron wavelength, $\lambda=4.5 \AA$, sample-to-detector distance $=955 \mathrm{~mm}$, sample and detector rotation angles: $\omega=5.5^{\circ}$ and $2 \theta=11^{\circ}$, acquisition time $=60 \mathrm{~min}$. D33 beamline $\left(5.0 \cdot 10^{-3}\right.$ $\AA^{-1}<q<0.3 \AA^{-1}$ ): neutron wavelengths: $\lambda_{1}=4.6 \AA$ and $\lambda_{2}=13.0 \AA$ sample-to-detector distances $=2 \mathrm{~m}, 10 \mathrm{~m}$, acquisition time $=10 \mathrm{~min}$ (in $\mathrm{D}_{2} \mathrm{O}$ ), $45 \mathrm{~min}$ (in MeOD-d4). For both spectrometers: neutron absorber: Boron Carbide (B4C), thickness of quartz Hellma cells: $1 \mathrm{~mm}$, temperature (controlled): $T=25^{\circ} \mathrm{C}$, background: $99.9 \% \mathrm{D}_{2} \mathrm{O}$. Direct beam, empty cell and $\mathrm{H}_{2} \mathrm{O}$ were also recorded for both spectrometers. The background sample $\left(D_{2} \mathrm{O}\right)$ signal was subtracted from the experimental data. $q$ is defined as $(4 \pi / \lambda) \sin \vartheta / 2$ where $\vartheta$ is the scattering angle between the incident and the scattered neutron beams. Data treatment was done with the home-made software package provided at the beamline. Direct determination of the number of neutrons in the incident beam and the detector cell solid angle allowed obtaining absolute values of the scattering intensity. The acquired 2D raw data were corrected for the scattering of the ambient background and the empty 
cell. Further, the data were normalized by the neutron flux on the samples to yield an absolute scale (cross section per unit volume). The data were radially averaged to yield the 1-D intensity distribution $I(q)$.

Fit of small angle scattering data (SAXS, SANS). All data presented were fitted using a classical core-shell sphere form factor model (assuming a unitary structure factor in the analyzed range of $q$-values), ${ }^{38}$ using the SasView 3.1.2 software (CoreShellModel) available free of charge on the developers' website. ${ }^{39}$ In the model, we consider the core to be composed of iron oxide and the shell of a homogeneous SL-NDA layer of variable thickness. One should be aware that this is a strong approximation, because SL-NDA could be described by at least three different regions of inequivalent densities: sophorose, oleic acid and NDA. Nonetheless, the goal of the fitting analysis in this work is not to precisely describe the morphology of the of the nanoparticle shell, but to demonstrate that SL-IONP have a shell with a thickness compatible with a monolayer of SL-NDA; the homogeneous core-shell model provides an adequate model with a minimum number of fitting variables to determine the presence and thickness of a reasonably homogeneous shell. All theoretical and technical details of both the model and fitting function can be 
found at on the developers' homepage ${ }^{39}$ The following parameters (Table 1) characterize the fitting function:

Table 1. Structural parameters used in the core-shell sphere model to fit both the SANS and SAXS data recorded for the SL-IONP.

\begin{tabular}{|c|c|c|c|}
\hline Parameter & Description & Fixed & Variable \\
\hline$d$ & Core diameter & $x$ & \\
\hline$t$ & Shell Thickness & & $x$ \\
\hline$\rho_{c}$ & Scattering Length Density (SLD) of the IONP core & $x$ & \\
\hline$\rho_{s}$ & Scattering Length Density (SLD) of the SL shell & & $x$ \\
\hline$\rho_{\text {solv }}$ & $\begin{array}{l}\text { Scattering Length Density (SLD) of the solvent }\left(\mathrm{H}_{2} \mathrm{O}\right. \\
\text { and } \mathrm{D}_{2} \mathrm{O} \text { for } \mathrm{X} \text {-ray and neutron scattering } \\
\text { experiments respectively) }\end{array}$ & $x$ & \\
\hline scale & Volume fraction & $\mathrm{x}$ & \\
\hline background & Background level & & $x$ \\
\hline PDI & Polydispersity & & $\mathrm{x}$ \\
\hline
\end{tabular}

The fixed parameters of the fitting function vary according to the type of technique used to study the samples. The fixed values which we have used in this work are reported 
hereafter. The nanoparticle diameter $d$ is determined independently by TEM and SAXS.

The SLD values have been determined using the SLD calculator tool in the SasView software. $\rho_{\text {solv }}(\mathrm{X}$-ray) $)=9.410 \times 10^{-6} \AA^{-2}$ and $\rho_{\text {solv }}$ (neutron $)=6.4 \times 10^{-6} \AA^{-2}$, where $\rho_{\text {solv }}(\mathrm{X}$ ray) and $\rho_{\text {solv }}$ (neutron) respectively refer to the X-ray and neutron SLD of $\mathrm{H}_{2} \mathrm{O}$ and $\mathrm{D}_{2} \mathrm{O} . \rho_{c}$ $(\mathrm{X}$-ray $)=40.0 \times 10^{-6} \AA^{-2}$ and $\rho_{c}$ (neutron $)=6.9 \times 10^{-6} \AA^{-2}$ refer to the X-ray and neutron SLD of iron oxide respectively, for which we have used the bulk density value of 5.17 g.cm ${ }^{-1}$. Polydispersity was allowed to vary between 0.1 and 0.15 and the volume fraction was set to $0.4 \%$ for samples $3.1 \mathrm{~nm}$ SL-IONP and $4.6 \mathrm{~nm} \mathrm{SL-IONP}$ and to $0.1 \%$ for sample $14.1 \mathrm{~nm}$ SL-IONP, the smaller value coming from the larger amount of SL-NDA in the $14.1 \mathrm{~nm}$ SL-IONP sample (please refer to main text for more details). In the final fitting procedure, $t$ and $\rho_{s}$ are the only structural variable parameters, while the background level is set as variable mainly to adjust the quality of the final fit. The typical size and composition of SL-NDA also imposes several constraints on the effective $t$ and $\rho_{s}$ values. One can reasonably consider $2.0<t(\mathrm{~nm})<3.5$ for a single SL-NDA layer, where the lower and upper limits consider plausible molecular bending and stretching; meanwhile, $9.6<\rho_{s}$ (X-ray) $\left(10^{-6} \AA^{-2}\right)<12$ and $1<\rho_{s}$ (neutron) $\left(10^{-6} \AA^{-2}\right)<5$ reasonable 
identify the range for both the X-ray and neutron shell SLD values, which can vary between a highly hydrated shell and a poorly hydrated organic layer.

\section{Results and Discussion}

IONP synthesis, ligand exchange and purification. Spherical, monodisperse and singlecrystalline iron oxide nanoparticles with diameters of $3.1 \pm 0.3 \mathrm{~nm}(\sigma=8.0 \%), 4.6 \pm 0.3$ $\mathrm{nm}(\sigma=7.0 \%)$ and $14.1 \pm 0.9 \mathrm{~nm}(\sigma=6.9 \%)$ were synthesized and characterized by HR- and LR-TEM. Figure S2 shows TEM micrographs of as-synthesized monodisperse oleic acid-coated iron oxide cores.

The exchange of hydrophobic oleic acid for sophorolipid-nitrodopamide ligand was performed using a method established earlier ${ }^{8}$ and yielded well-dispersed SL-NDA coated IONP (SL-IONP) with three different core sizes (Figure 1). Single-crystallinity of the IONP cores was revealed by HR-TEM (inset in Figure $1 \mathrm{C}$ and Figure S3) and the lattice spacing in the (220) direction was measured to be $0.29 \mathrm{~nm}$. The electron diffraction pattern (inset Figure 1B) shows the high crystallinity of the IONP. The determined ratios of $d$-spacings show good agreement with the JCPDS database values given for 
maghemite or magnetite. ${ }^{40}$ Single-crystalline iron oxide nanoparticles (magnetite and maghemite) in this size-range are known to be superparamagnetic, thereby possessing attractive properties for biomedical imaging and drug delivery.
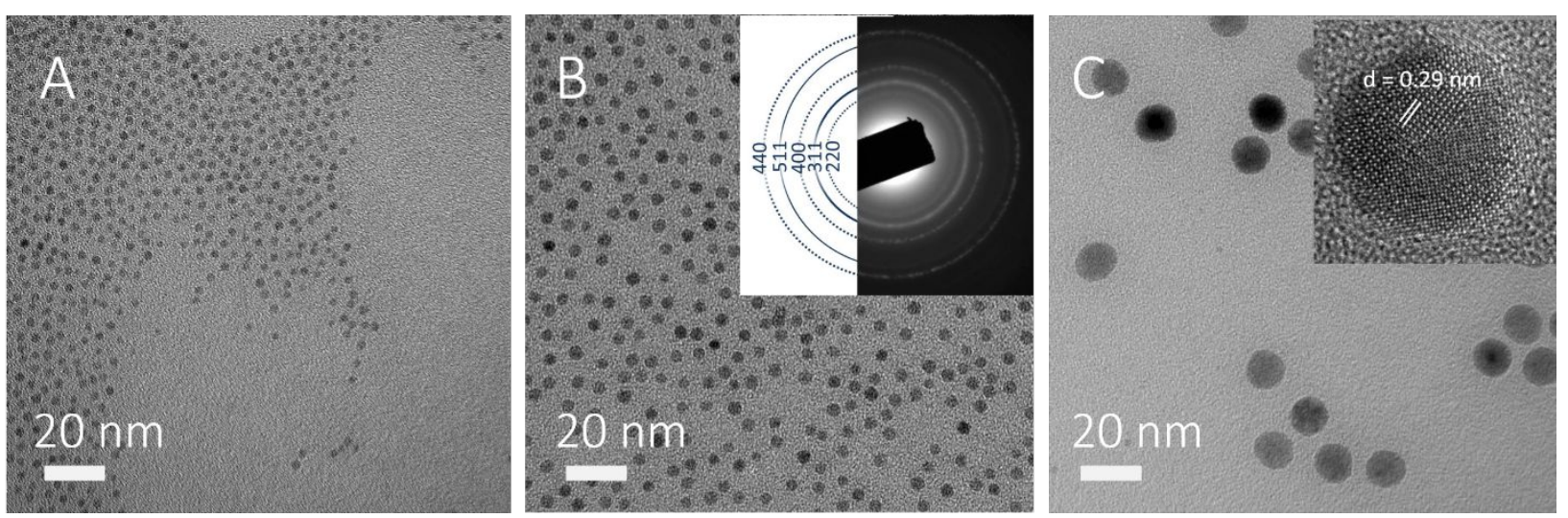

Figure 1. TEM micrographs of three sizes of SL-IONP of sizes A) $3.1 \pm 0.3 \mathrm{~nm}, \mathrm{~B}) 4.6 \pm$ $0.3 \mathrm{~nm}$ and C) $14.1 \pm 0.9 \mathrm{~nm}$. The insets in B) and C) show the electron ring diffraction pattern and a high-resolution micrograph of $14.1 \mathrm{~nm}$ IONP, revealing the high crystallinity of the single crystal cores.

To demonstrate colloidal stability of SL-grafted IONP, excess ligand should be removed from the nanoparticle dispersion. Thus, SL-IONP were purified using two common 
purification methods for nanoparticles, namely dialysis and membrane centrifugation to remove the excess of the micelle-forming glycolipid ligand.

The functionalization of the IONP with SL-NDA through ligand replacement was first evaluated using ATR-FTIR. Figure 2 shows the FTIR spectra of the compounds and products. NDA has a characteristic C-O stretch band at $1272 \mathrm{~cm}^{-1},{ }^{41}$ which can be found in the product of the purified SL-NDA but not in pure SL. Likewise, the "sugar bands" corresponding to C-O and C-O-C stretch modes at $1021 \mathrm{~cm}^{-1}$ and $1069 \mathrm{~cm}^{-1},{ }^{42}$ respectively, from the sophorolipid are also strong in the SL-NDA product. Finally, the bands of the amide bond formed between SL and NDA are clearly visible in SL-NDA. Figure 2 shows that two characteristic bands of oleic acid, the $\mathrm{C}=\mathrm{O}$ stretch at $1707 \mathrm{~cm}^{-1}$ (which is slightly higher than for sophorolipid) and the out-of-plane O-H stretch at 963 $\mathrm{cm}^{-1}$ are absent or insignificant compared to the characteristic bands of the sophorolipid and NDA. ${ }^{32}$ In summary, the ATR-FTIR results strongly support successful ligand replacement of the oleic acid by SL-NDA to form iron oxide nanoparticles with a shell of sophorolipid, strongly linked by nitrodopamide. 


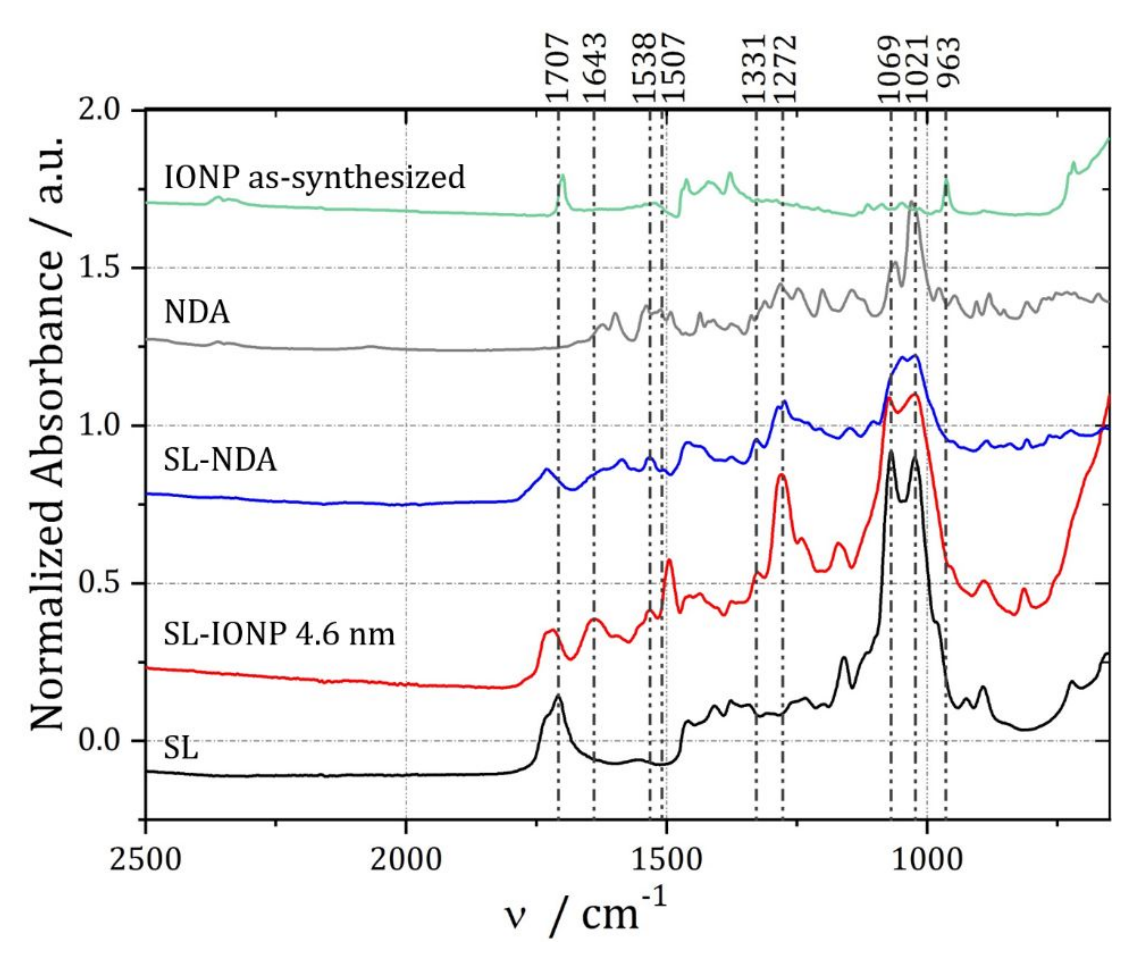

Figure 2 ATR-FTIR spectra of as-synthesized IONP (oleic acid capped, green), nitrodopamine (grey), sophorolipid-NDA (blue), SL-IONP $4.6 \mathrm{~nm}$ (red) and sophorolipid (black). Curves have been shifted vertically for clarity. With attributions: $\delta_{(C=O)-O H}$ (oleic acid): $963 \mathrm{~cm}^{-1}, v_{C-O}$ (sugar): 1021 and $1069 \mathrm{~cm}^{-1}, v_{\mathrm{C}-\mathrm{O}}$ (catechol): $1272 \mathrm{~cm}^{-1}, v_{s}\left(\mathrm{NO}_{2}\right)$ : $1331 \mathrm{~cm}^{-1}, v_{\mathrm{C}-\mathrm{C}}\left(\right.$ catechol): $1507 \mathrm{~cm}^{-1}, v_{a}\left(\mathrm{NO}_{2}\right): 1538 \mathrm{~cm}^{-1}, v_{\mathrm{C}=\mathrm{O}}$ (amide): $1643 \mathrm{~cm}^{-1}$, $v_{\mathrm{C}=\mathrm{O}}\left(\right.$ amide) and $\delta_{\mathrm{N}-\mathrm{H}}$ (amide): $1538 \mathrm{~cm}^{-1}$ and,$v_{\mathrm{C}=\mathrm{O}}$ (carboxylic): $1707 \mathrm{~cm}^{-1} .41-43$ 
The average grafting density of SL-NDA ligand irreversibly bound to the Fe-ions at the IONP surface was evaluated after purification by TGA. Typical TGA weight loss profiles for $3.1 \mathrm{~nm}$ SL-IONP, $4.6 \mathrm{~nm}$ SL-IONP and $14.1 \mathrm{~nm}$ SL-IONP are shown in Figure S4. The total organic content (TOC) was determined as the fraction of mass loss up to $650^{\circ} \mathrm{C}$. If all excess ligand is removed, the TOC can be converted into the dispersant grafting density using the known molecular weight of SL-NDA, the defined average iron oxide core area determined by TEM and a known iron oxide core density of $5.17 \mathrm{~g} / \mathrm{cm}^{3}$. We thus assume for this calculation that all oleic acid was replaced by SL-NDA during the rigorous ligand replacement and purification. Earlier studies have shown that ligand replacement of OA by hydrophobic ligands is challenging and can leave significant residues within the nanoparticle shell. ${ }^{32,} 44$ If this is the case, the amount of SL-NDA grafted to the IONP would be overestimated. However, we have previously investigated the ligand replacement and purification protocol used here and shown that the amount of residual OA is below the detection limit by ATR-FTIR. As discussed above, the ATR-FTIR data shown in Figure 2 supports this finding. Additionally, the calculation of the grafting density based on the TGA data can yield a higher value than the actual grafting density if there 
is also free ligand present in a sample. We therefore list the average number of ligands ( $n_{\text {ligands }}$ ) per $\mathrm{nm}^{2}$ in Table 2 calculated in the same way for differently sized IONP purified by dialysis or membrane centrifugation (Amicon $50 \mathrm{kDa} \mathrm{MWCO}$ ) to take into account the possible presence of free ligand.

Table 2. TGA results for SL-IONP of three different core sizes. Total organic contents (TOC) was converted into average number of ligands $/ \mathrm{nm}^{2}\left(\mathrm{n}_{\text {ligands }} / \mathrm{nm}^{2}\right)$ using the known SL-NDA Mw (902 g/mol).

\begin{tabular}{|c|c|c|}
\hline SL-IONP type & $n_{\text {ligands }} / \mathrm{nm}^{2}$ [dispersant $/ \mathrm{nm}^{2}$ ] & $\begin{array}{l}\text { TOC } \\
{[\% \mathrm{w} / \mathrm{w}]}\end{array}$ \\
\hline $\begin{array}{lccc}3.1 \pm & \pm & 0.3 & \mathrm{~nm} \\
\text { dialyzed } & & \end{array}$ & $8.0^{\mathrm{a}}$ & $82.3^{a}$ \\
\hline $3.1 \pm 0.3 \mathrm{~nm}$ Amicon & $7.1^{\mathrm{a}}$ & $80.5^{a}$ \\
\hline $\begin{array}{lccc}4.6 \quad \pm & 0.3 & \mathrm{~nm} \\
\text { dialyzed } & & \end{array}$ & 4.3 & 60.8 \\
\hline $4.6 \pm 0.3 \mathrm{~nm}$ Amicon & 3.3 & 55.6 \\
\hline $\begin{array}{lll}14.1 \quad \pm & 0.9 & \mathrm{~nm} \\
\text { dialyzed } & & \end{array}$ & $37.4^{a}$ & $82.1^{a}$ \\
\hline
\end{tabular}



$14.1 \pm 0.9 \mathrm{~nm}$ Amicon
$45.2^{\mathrm{a}}$
$84.8^{a}$

a Samples contained large amounts of excess ligand.

The limiting factor for ligand adsorption to the IONP surface is given by the number of surface iron sites (3.5 - 5.9 depending on crystal plane $)^{45}$ or the spatial requirements (footprint) of the ligand. The hydrated sugar headgroup and the unsaturated fatty acid structure of SL could sterically hinder achieving the maximum grafting density of NDA, but this steric blocking is reduced by the high curvature of the IONP that provides much larger volume for the headgroup than for the binding group. The grafting density of the deacetylated acidic form of sophorolipids (the same used in this work) varies according to the system under study; one finds between 2 and 3 molecules $/ \mathrm{nm}^{2}$ on a flat gold surface (evaluated by X-ray photoelectron spectroscopy and quartz crystal microbalance with dissipation monitoring), ${ }^{46} 0.96$ molecules $/ \mathrm{nm}^{2}$ at the air/water interface (surface tension) $)^{47}$ and between 0.5 and 1.0 molecules $/ \mathrm{nm}^{2}$ in micelles respectively at the micelle/water and alkyl/sophorose interface (evaluated by SAXS)..$^{20}$ The SL-IONP with a diameter of $4.6 \mathrm{~nm}$ purified by Amicon have a ligand density of 3.3 molecules $/ \mathrm{nm}^{2}$, which 
is within the range of plausible grafting densities taking both the density of grafting sites and steric blocking during grafting into account. The other systems display values of SL$\mathrm{NDA} / \mathrm{nm}^{2}$ that are higher than the number of possible grafting sites on the IONP surface, which sets an absolute upper bound on the grafting density. Hence, the observed values above 5 molecules $/ \mathrm{nm}^{2}$ (Table 2) must be due to excess ligand that is not grafted to the IONP surface. The excess ligand could not be removed with Amicon filters and dialysis could also not reduce the organic content of the samples. It has been reported that sophorolipids form micellar structures in water, ${ }^{20}$ and cryo-TEM micrographs in Figure $3 \mathrm{~A}$ and Figure S5 demonstrate that also SL-NDA self-assembles into both spherical and filamentous structures. Since it is not a goal of this work to describe the self-assembly properties of SL-NDA in water, we prefer not to go into descriptive details; we will simply refer to a general micellar and filamentous self-assembled phase of SL-NDA in coexistence with SL-IONP.

Figures 3B-C and Figures S6 and S7 show cryo-TEM images of 14.1 and $4.6 \mathrm{~nm}$ SLIONP purified by membrane centrifugation. The $14.1 \mathrm{~nm}$ cores have the highest organic content according to TGA, while the $4.6 \mathrm{~nm}$ cores have an organic content compatible 
with maximum grafting of SL-NDA to the IONP surface. In addition to the nicely dispersed SL-IONP with high electron density contrast, large and elongated structures with lower contrast are present primarily in the $14.1 \mathrm{~nm}$ core sample; these strongly resemble the filamentous structures formed by SL-NDA in water, depicted in Figure 3A.
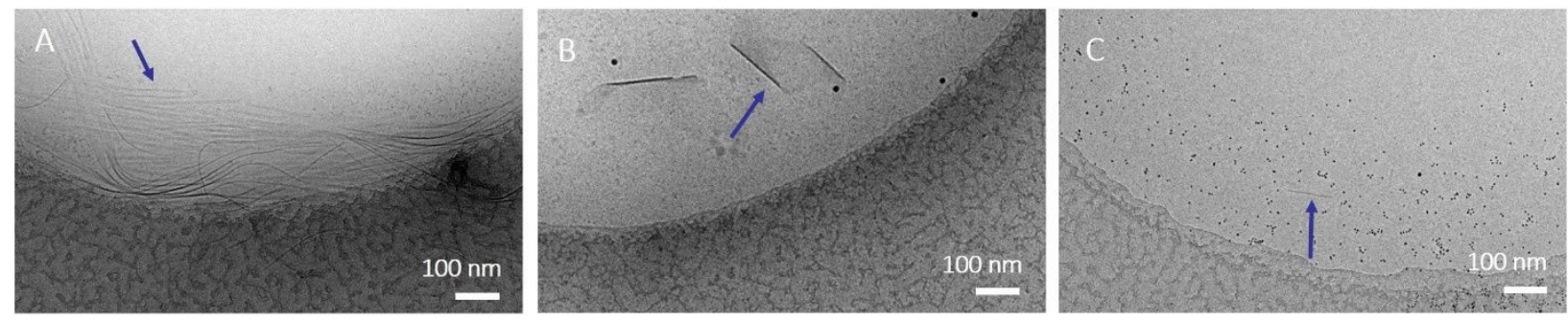

Figure 3. Cryo-TEM micrographs in $\mathrm{H}_{2} \mathrm{O}$ of A) SL-NDA, B) $14.1 \mathrm{~nm}$ SL-IONP and C) 4.6 nm SL-IONP. The blue arrows indicate lamellar structures formed by excess ligand; the black dots are nanoparticles (see Figure S6 and S7 for enlarged pictures).

This feature suggests why SL-NDA could not be fully removed by dialysis or by membrane centrifugation: the cut-off pore size of the membranes is larger than a single SL-NDA molecule, but smaller than their self-assembled phase. Dialysis should in 
principle allow for removal of excess lipid from the sample, but in practice, selfaggregating molecules with a low critical aggregation constant or very slow kinetics of desorption (low $k_{\text {off }}$ ) will make dialysis very inefficient; this is apparently the case for dialysis of SL-NDA forming large and stable structures. An attempt to improve the dialysis with salt $(\mathrm{NaCl})$ by shifting the critical micelle concentration failed for this system since SL-NDA micelles are insensitive to salt (cf. SANS data below). Interestingly, the small core sample $(4.6 \mathrm{~nm})$ had much less excess ligand after purification than the large core sample. This could tentatively be explained by that for the larger cores a larger excess of SL-NDA to IONP surface area was used, which favors growth of more and larger micelles during grafting or during resuspension. The $4.6 \mathrm{~nm}$ SL-grafted IONP presumably had a lower amount of excess SL-NDA in solution after grafting and consequently a negligible excess of SL-NDA in the dispersion after purification. This interpretation of the purification and TGA data is supported by cryo-TEM (Figure $3 \mathrm{C}$ and Figure S7), in which many stabilized nanoparticles are observed, but only a limited amount of SL-NDA. With the difficulty to purify nanoparticles from SL-NDA micelles and filaments, one can try to minimize the excess of SL-NDA during grafting to suppress micelle formation. However, 
this easily leads to inefficient grafting and sub-monolayer dispersant grafting density on the particle surface. Grafting with large SL-NDA excess is therefore recommended for applications where excess micelles can be tolerated but particle colloidal stability is of paramount importance.

\section{Characterization of the SL-NDA ligand shell with SANS and SAXS. It is typically} assumed that the high colloidal stability of coated nanoparticles is explained by the presence of a single layer of ligand; this argument may also hold for the SL-IONP presented here. However, most classical techniques, like DLS or TEM, on which this assumption is based, are generally not providing in situ or detailed enough data to quantitatively investigate the presence, structure and properties of the shell in support of this hypothesis. SAXS, which is sometimes used for this purpose, is mostly not suitable for hybrid inorganic-organic nanoparticles, because the contrast between the solvent and the electron-rich inorganic core generally masks the signal of the shell resulting from the poor contrast between the solvent and the shell. The combined use of X-ray and neutron scattering is the only method to probe the structural characteristics such as the thickness 
of the nanoparticle organic shell. In fact, neutrons, being scattered by the atom nuclei, are less sensitive to atoms with high atomic number. We therefore combined SAXS and SANS as a reliable method to estimate the extension of the SL-NDA shell for SL-IONP purified by Amicon filtration, that had a low excess of ligand in micelles.

SAXS was used to determine and confirm the size of the IONP cores. Under X-ray irradiation, the inorganic core of SL-IONP nanoparticles has a very high contrast $\left(\rho_{\text {solv }}-\right.$ $\rho_{c}=-30.60 \times 10^{-6} \AA^{-2}$, Table 3) if compared to the shell-solvent $\left(\rho_{\text {solv }}-\rho_{s}=-0.57 \times 10^{-6}\right.$ $\AA^{-2}$, Table 3); for this reason, SAXS only provides structural information on the nanoparticle cores. Figure 4A (green circles) shows the SAXS profile for $4.6 \mathrm{~nm}$ SL-IONP. The best fit using a core-shell sphere form factor can be obtained using an average nanoparticle core diameter of $4.8 \pm 0.4 \mathrm{~nm}$ and a null value for the shell thickness. This is in very good agreement with the diameter obtained by TEM $(4.6 \pm 0.3 \mathrm{~nm})$ within the experimental errors. The corresponding SANS pattern (Figure 4A, blue circles) is shifted towards the low- $q$ region, which signifies sensitivity to the larger size resulting from the much higher contrast of neutrons to the lipid shell. 
The SANS pattern can be modeled using an imposed (from SAXS data) core diameter of $d=4.8 \pm 0.4 \mathrm{~nm}$ and a shell thickness of $t=2.5 \pm 0.2 \mathrm{~nm}$ obtained by fitting a coreshell model to the scattering data. This simple model assumes a uniform scattering length density throughout the shell, which is clearly a simplification, but it can be used to estimate the shell thickness by fitting the shift of the first minimum of the form factor. The calculated length of SL, considering the $120^{\circ}$ angle due to the mono-unsaturation, can be estimated to $2.6 \mathrm{~nm}$, where $1.6 \mathrm{~nm}$ are attributed to the fatty $\operatorname{acid}^{48}$ and $1 \mathrm{~nm}$ to sophorose, in analogy to the size of maltose, a common disaccharide. ${ }^{49}$ The size of NDA is estimated to about $1 \mathrm{~nm} \cdot{ }^{50}$ Under these conditions, the maximum expected size for SL-NDA is about $3.6 \mathrm{~nm}$. The modeled thickness of $t=2.5 \pm 0.2 \mathrm{~nm}$ is shorter than the maximum monolayer thickness and demonstrates that the shell of monodisperse SL-IONP is comprised of a single layer of SL-NDA of less than maximum density. From the fit, one can also evaluate an average shell scattering length density of $\rho_{s}=3.39 \times 10^{-6} 10^{-6} \AA^{-2}$, a value which is reasonable for a hydrated (in $\mathrm{D}_{2} \mathrm{O}$ ) organic lipid layer. ${ }^{51}$ The lower density or slightly lower thickness that is observed can be explained in several ways. One can, e.g., consider that the shell could contain other smaller ligands than SL-NDA, such as 
residual catalyst (COMU), oleic acid or unreacted NDA or SL. The combination of TGA, FTIR and NMR data make this hypothesis unlikely. COMU, which was observed as residue in the SL-NDA batches used for grafting the NPs, has very low affinity for the iron oxide surface. All techniques employed show no evidence of residual OA, while ${ }^{1} \mathrm{H}$ NMR shows that the SL-NDA does not contain free NDA. A residual amount of unreacted SL (10-20 mol\% with respect to SL-NDA) is present. The carboxyl endgroup of SL has high affinity to the IONP, but NDA functionalized ligands possess much higher affinity and outcompete carboxyl anchored ligands on the surface. This is demonstrated by FTIR showing the successful ligand replacement of OA by SL-NDA and previous studies of e.g. palmityl-NDA ligand replacement of OA. ${ }^{32}$ Furthermore, our original attempts to replace OA with SL failed to produce water-soluble particles, strongly suggesting that replacement of OA will be driven by SL-NDA. Thus, although our data do not allow its exclusion, we do not expect significant amounts of unmodified SL on the IONP.

A more plausible explanation for the thin shell is that the SL-NDA is grafted on a highly curved surface, which provides a rapidly increasing conical volume to the SL-NDA even at the highest grafting density. ${ }^{52}$ This allows for a large tilt or bending of the molecules, 
i.e. a lower effective shell thickness. The extended length of $S L$ is calculated according to the classical Tanford formula. However, SL is known to adopt a bent configuration, which yields an effectively thinner layer than theoretically calculated. For instance, XPS has shown that a single self-assembled monolayer of cysteamine-modified SL grafted onto flat gold has a thickness of only $3.0 \pm 0.2 \mathrm{~nm},{ }^{42}$ while small-angle X-ray and neutron scattering measurements showed that the total radius of an acidic SL micelle is generally below $4 \mathrm{~nm}$, i.e, approximately the size of a single SL molecule. ${ }^{53-54} \mathrm{~A}$ layer grafted on a highly curved NP would be expected to yield a thickness in between these estimates.

Finally, one should not exclude that the simplification of assuming a uniform scattering length density, which could be invalidated by the conical ligand volume and the highly hydrated headgroup, would also lead to a fit predicting an effectively thinner shell. Whichever the dominant of the two latter explanations, the SAXS/SANS data demonstrate a single thin SL-NDA layer serving as the stabilizing shell on the IONP. 

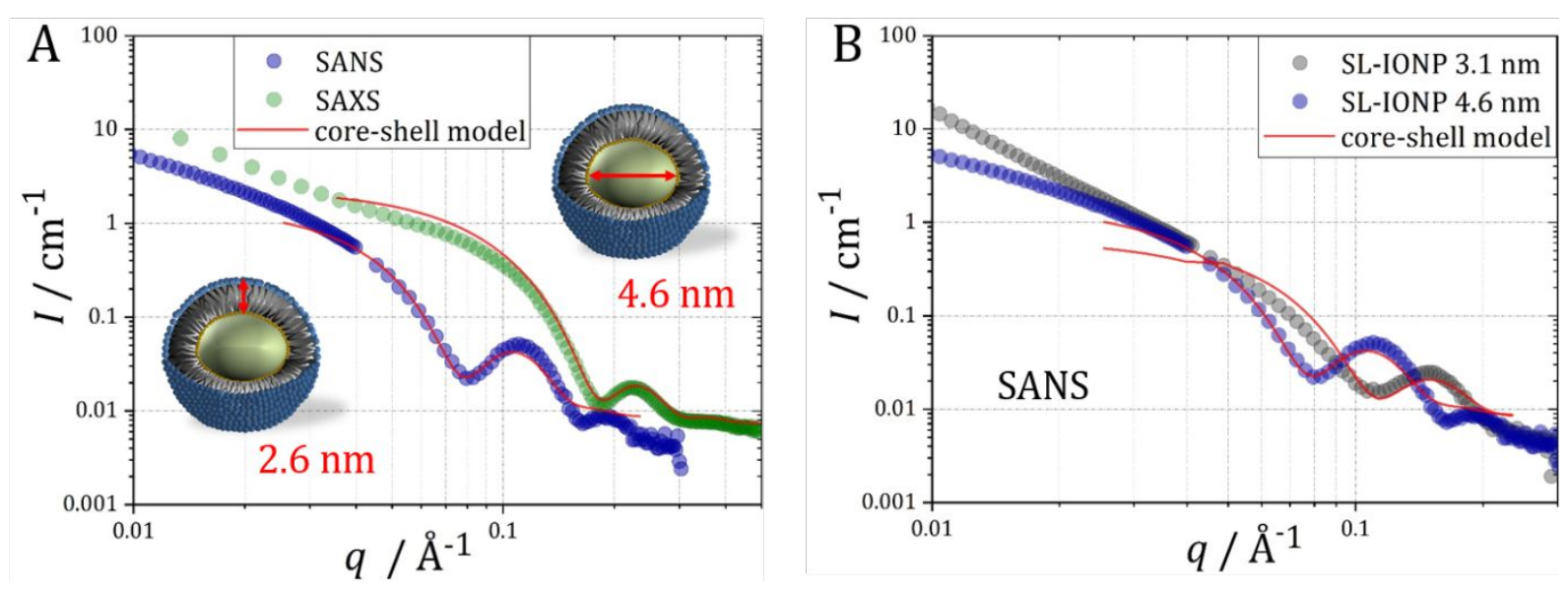

Figure 4. A) SANS (blue) and SAXS (green) profiles for $4.6 \mathrm{~nm}$ SL-IONP in $\mathrm{D}_{2} \mathrm{O}$. The red lines show the fit obtained from a core-shell sphere model. The best fit for SAXS was obtained using a core diameter of $4.8 \mathrm{~nm}$ and a null value for the shell. B) Comparison of the SANS data of $4.6 \mathrm{~nm}$ (blue) and $3.1 \mathrm{~nm}$ SL-IONP (grey). As expected, the profile for smaller SL-IONP is shifted to larger $q$. The red lines show the fit obtained with a homogeneous core-shell model.

Similar results are obtained for the smaller $3.1 \mathrm{~nm}$ SL-IONP (Figure 4B). The SANS pattern can be modelled with a fixed (from SAXS data) core diameter, $d=3.0 \pm 0.2 \mathrm{~nm}$ and a fitted shell thickness of $t=2.2 \pm 0.2 \mathrm{~nm}$. These data are summarized in Table 3 , 
which also contains the solvent-core and solvent-shell scattering length density contrasts fitted from the SANS data: $\rho_{\text {solv }}-\rho_{c}=-0.57 \times 10^{-6} \AA^{-2}$ while $\rho_{\text {solv }}-\rho_{S}$ is in the order of 3.0 $x 10^{-6} \AA^{-2}$. This demonstrates that the shell has higher contrast than the iron oxide core, and therefore dominates the scattering signal of the core-shell particle. The absolute value of the shell scattering length density for $3.1 \mathrm{~nm}$ SL-IONP is $\rho_{s}=3.45 \times 10^{-6} 10^{-6} \AA^{-2}$ and in good agreement with what is found for the $4.6 \mathrm{~nm}$ core SL-IONP.

Table 3. Structural parameters for the $3.1 \mathrm{~nm}$ and $4.6 \mathrm{~nm}$ SL-IONP obtained by combining TEM, SAXS and SANS analyses.

\begin{tabular}{|l|l|l|l|l|l|}
\hline \multirow{2}{*}{ Sample } & Technique & $\begin{array}{l}\text { Core diameter } \\
d(\mathrm{~nm})\end{array}$ & $\begin{array}{l}\text { Shell Thickness } \\
t(\mathrm{~nm})\end{array}$ & $\begin{array}{l}\rho_{\text {solv }}-\rho_{c} \\
\left(\times 10^{-6} \AA^{-2}\right)\end{array}$ & $\begin{array}{l}\rho_{\text {solv }}-\rho_{s} \\
\left(\times 10^{-6} \AA^{-2}\right)\end{array}$ \\
\hline \multirow{3}{*}{$\begin{array}{l}3.1 \quad \mathrm{~nm} \\
\text { SL-IONP }\end{array}$} & TEM & $3.1 \pm 0.2$ & - & - & - \\
\cline { 2 - 6 } & SAXS & $3.0 \pm 0.4$ & - & -30.60 & $<-1.00$ \\
\cline { 2 - 6 } & SANS & $3.0 \pm 0.4$ & $2.5 \pm 0.2$ & -0.57 & 2.63 \\
\hline \multirow{3}{*}{$\begin{array}{l}4.6 \quad \mathrm{~nm} \\
\text { SL-IONP }\end{array}$} & TEM & $4.6 \pm 0.2$ & - & - & - \\
\cline { 2 - 6 } & SAXS & $4.8 \pm 0.4$ & - & -30.60 & $<-1.00$ \\
\cline { 2 - 6 } & SANS & $4.8 \pm 0.4$ & $2.2 \pm 0.2$ & -0.57 & 3.00 \\
\hline
\end{tabular}


The scattering curve of the $14.1 \mathrm{~nm}$ SL-IONP sample was radically different to those of the dispersions of the two smaller SL-grafted IONP and is shown in Figure 5. The typical SANS spectrum for $14.1 \mathrm{~nm}$ SL-IONP in $\mathrm{D}_{2} \mathrm{O}$ (blue filled circles) is relatively featureless in $\mathrm{D}_{2} \mathrm{O}$, but it shows strong scattering with a characteristic slope in the low- $q$ region that is not present in the SANS spectra of the smaller SL-grafted IONP. This additional feature can be explained by comparing the data to the SANS spectrum of the pure SL-NDA ligand solution (Figure 5, grey filled circles), which nicely matches the spectrum of the $14.1 \mathrm{~nm}$ SL-IONP sample. Thus, in good agreement with the TGA data presented in Figure S4 and Table 2, the SANS signal of the $14.1 \mathrm{~nm}$ SL-IONP dispersion is dominated by SLNDA in the form of filamentous structures instead of the core-shell particles. The higher contrast of SL-NDA to $D_{2} \mathrm{O}$ than of iron oxide to $\mathrm{D}_{2} \mathrm{O}$ further contributes to the dominance of scattering from the SL-NDA micelles. These micelles were also prevalent in cryo-TEM images of the $14.1 \mathrm{~nm}$ SL-IONP sample (see Figure 3B). Nonetheless, SL-NDA is soluble in perdeuterated methanol, as shown by the corresponding poor SANS signal (grey empty 
circles) in Figure 5 5. As expected, the SANS pattern of $14.1 \mathrm{~nm}$ SL-IONP (blue empty circles) in MeOD-d4 loses the SL-NDA scattering profile and it rather presents the typical feature of spherical objects. A shell-free $14.1 \mathrm{~nm}$ diameter IONP nanoparticle would be expected to have the first minimum of the form factor around $q=0.4 \mathrm{~nm}^{-1}$, whereas the minimum of the oscillation of the form factor measured for the $14.1 \mathrm{~nm}$ SL-IONP falls between $0.2 \mathrm{~nm}^{-1}<q<0.3 \mathrm{~nm}^{-1}$, suggesting a spherical object sized between 20 and 30 $\mathrm{nm}$. This is largely in agreement to what would be expected for a $14.1 \mathrm{~nm}$ iron oxide core, stabilized by a single SL-NDA layer even if the conditions of analysis (excess of SL-NDA, methanol) and likely presence of aggregates do not allow the quantification of the thickness of the SL-NDA layer. 


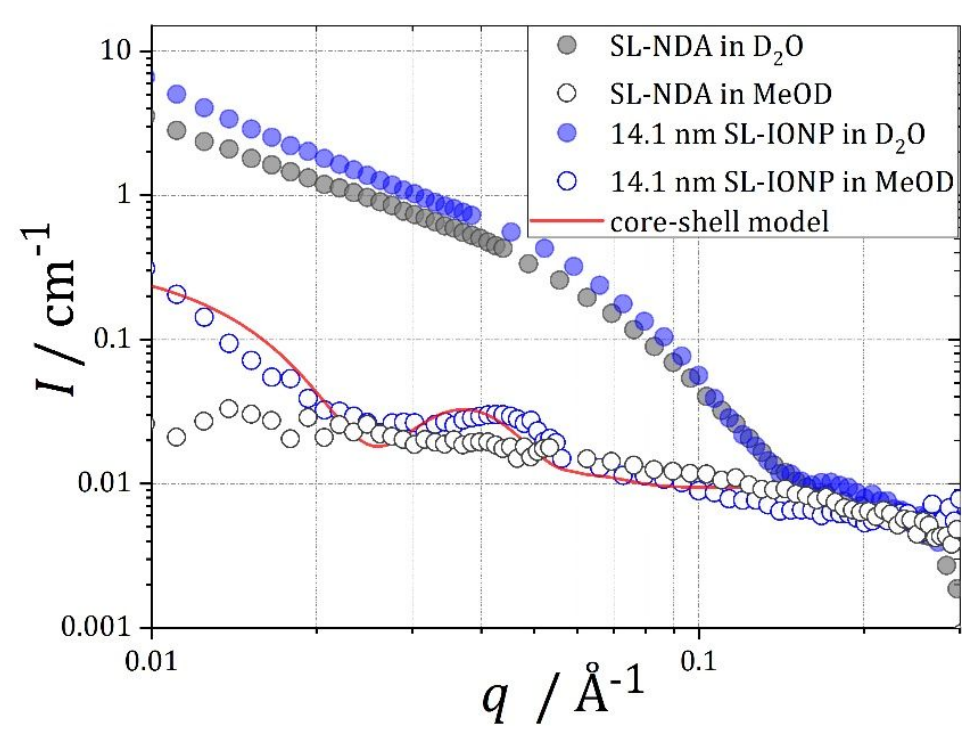

Figure 5. SANS data recorded for $14.1 \mathrm{~nm}$ SL-IONP in $\mathrm{D}_{2} \mathrm{O}$ (blue filled circles) and MeOD$\mathrm{d} 4$ (blue empty circles) and the pure ligand SL-NDA in $\mathrm{D}_{2} \mathrm{O}$ (grey filled circles) and MeODd4 (grey empty circles).

Colloidal stability and long-term stability. Removal of all monomeric free SL-NDA, is a good test of the colloidal stability of SL-NDA-grafted IONP. However, a biological environment is much more challenging for IONP colloidal stability, due to the higher concentration of biological colloids, displaying a larger variety of attractive interactions and leading to nanoparticle aggregation. ${ }^{5}$ Thus, colloidal stability of nanoparticles meant 
to perform functions in biotechnological or medical applications should be tested under conditions where they are exposed to full physiological ionic strength as well as to concentrations of protein similar to those that could be encountered during application. Therefore, we suspended the SL-IONP in $10 \%$ fetal calf serum (FCS), which has a lower concentration than full serum, but at which the colloidal stability can be monitored by DLS. $10 \%$ serum also corresponds to the typical additive to cell culture media. We performed the measurements while heating from $20{ }^{\circ} \mathrm{C}$ to $70{ }^{\circ} \mathrm{C}$ and back. These conditions are known to weaken the hydration of the nanoparticle shell ${ }^{55}$ and thereby reduce the colloidal stability and decrease the ability to suppress adsorption of serum protein. Adsorption of denatured proteins leads to irreversible aggregation and precipitation of insufficiently stabilized nanoparticles ${ }^{56}$. Figure 6 shows the evolution of the volume weighted hydrodynamic diameter $D_{H}$ of $4.6 \mathrm{~nm} \mathrm{SL-IONP}$ in water with $10 \% \mathrm{FCS}$ with temperature. Volume weighed $D_{H}$ of $4.6 \mathrm{~nm}$ SL-IONP in water is shown in Figure S8A. The hydrodynamic diameter of $4.6 \mathrm{~nm}$ SL-IONP was measured to be around $12 \mathrm{~nm}$, which was stable after more than 1.5 years in storage. The SL-IONP with $3.1 \mathrm{~nm}$ and $14.1 \mathrm{~nm}$ core sizes appeared equally stable in dispersion over the same time period. However, the 
stability could not be quantitatively confirmed by the hydrodynamic diameter, as there were excess SL-NDA in these samples. All investigated SL-IONP have small diameters in the superparamagnetic range and they are stabilized predominantly by steric and hydration forces from the hydrophilic headgroup region of the sophorolipid. It is advantageous for many applications to have a thin stabilizing shell of this kind to reduce the overall hydrodynamic size. However, we note that the strength of the long-range attractive van der Waals forces increases if the inorganic nanoparticle core is made larger. It is likely that there is a maximum core size for which an SL-NDA shell can provide sufficient stabilization against aggregation by dipolar interactions.

The hydrodynamic diameter of the $4.6 \mathrm{~nm}$ SL-IONP is in reasonable agreement with the size of the core-shell nanoparticle determined by SANS, $\sim 9.2 \mathrm{~nm}$, which might underestimate the steric or hydrodynamic size due to the fitting of a homogeneous shell. The DLS result is also in good agreement with a theoretical size of a core-shell IONP calculated from the diameter of the core measured by TEM $(4.6 \mathrm{~nm})$ and the length of a SL-NDA molecule ( $3 \mathrm{~nm}$ ), which implies a total steric diameter of $10.6 \mathrm{~nm}$ diameter. Thus, both DLS and SANS data strongly suggest a monolayer of SL-NDA ligands on the NP 
surface. The size distribution measured for the monodisperse iron oxide nanoparticles grafted with SL-NDA is narrower and with a smaller $D_{H}$ than the SL-coated iron oxide nanoparticles reported in our earlier work. ${ }^{26}$ This is attributed to the novel synthesis protocol with improved ligand and anchor chemistry for grafting to monodisperse iron oxide core particles. Volume weighted $D_{H}$ size distributions of $10 \%$ FCS in Milli-Q without nanoparticles measured at $20^{\circ} \mathrm{C}$ and $70{ }^{\circ} \mathrm{C}$ are shown in Figure S8B. The size of serum proteins was measured to be $\sim 5 \mathrm{~nm}$ at $20{ }^{\circ} \mathrm{C}$. A size shift to $8 \mathrm{~nm}$ was observed at elevated temperatures.
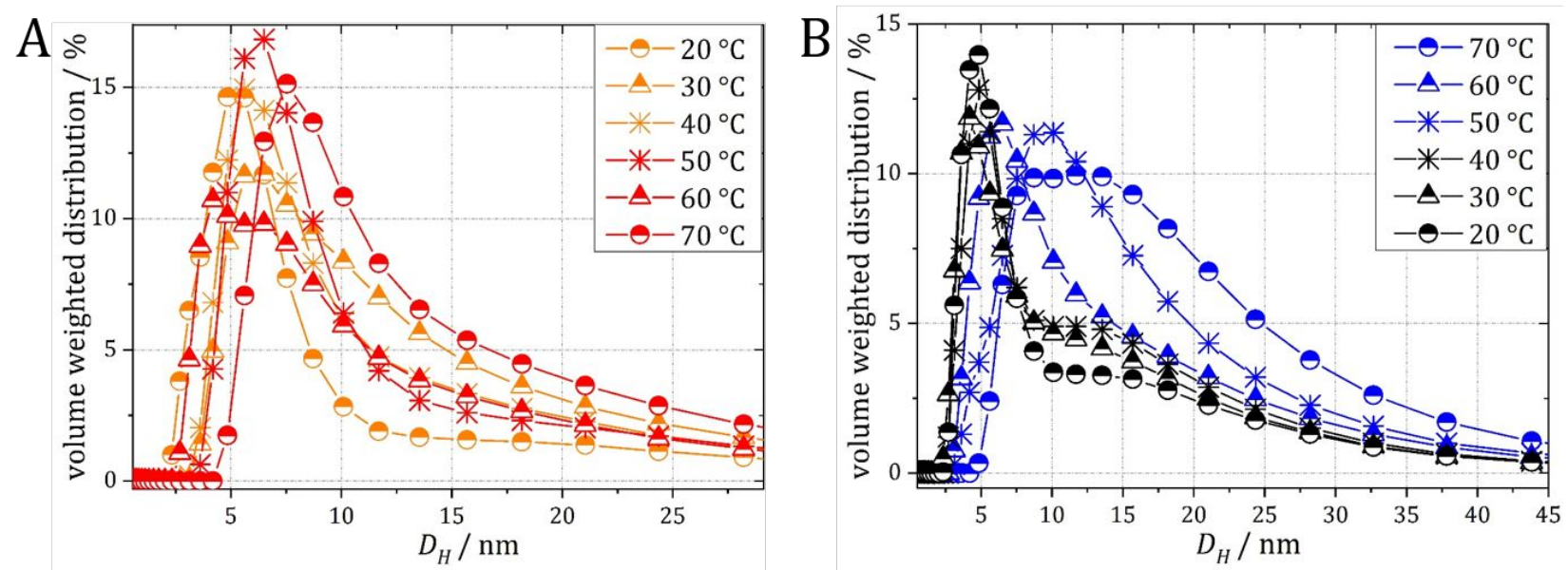

Figure 6. Volume weighted hydrodynamic diameters $D_{H}$ of $4.6 \mathrm{~nm}$ SL-IONP dispersed in water with $10 \% \mathrm{FCS}$ heated from A) $20^{\circ} \mathrm{C}$ to $70{ }^{\circ} \mathrm{C}$ and B) cooled from $70{ }^{\circ} \mathrm{C}$ to $20^{\circ} \mathrm{C}$ with 3 minutes equilibration time at each temperature. 
When heating up the SL-IONP in FCS, we observed a size distribution with the main peak corresponding to the abundant serum albumin, with a tail towards larger sizes contributed by the SL-IONP. Upon heating, the fraction of small objects (serum albumin) in the size distribution decreased and the volume fraction of larger objects (nanoparticles) increased. This results from partial denaturation and aggregation of serum proteins. This trend becomes even more obvious in Figure $6 \mathrm{~B}$ where a temperature of $70{ }^{\circ} \mathrm{C}$ was held for 8 minutes to allow for more equilibration of the sample. Here, the apparent volume fraction of nanoparticles is even larger, which could be due to precipitation of serum protein. Importantly, no aggregation and precipitation of SL-IONP was observed at any temperature, with or without exposure to denatured serum protein. This can also be observed from the intensity weighted size distributions for the same temperature cycling experiment (Figure S11). Figure S9 shows a photograph of SL-IONP after temperature cycling. Aggregation of SL-IONP could not be induced by heating the samples in FCS at $70^{\circ} \mathrm{C}$ for $3 \mathrm{~h}$ (Figure S10). Similar to the observations during temperature cycling, only a 
small shift in size is observed after heating for $3 \mathrm{~h}$ and a very small peak in the intensity weighted $D_{H}$ appears at large sizes, indicating the aggregation of the protein that precipitated after $3 \mathrm{~h}$ heating (Figure S12). These data, showing the excellent stability of SL-IONP with temperature, can be explained by the small influence that temperature has on the dehydration of the carbohydrate moiety of sugar-based capping agents. Sugarbased dispersants are both hydrogen-bond donors and acceptors, which allows for a bulk water-like hydrogen-bonding network and thereby low sensitivity to dehydration upon increasing temperature.

The excellent colloidal stability of the SL-IONP nanoparticles is further demonstrated by additional experiments performed in the presence of $1 \mathrm{M} \mathrm{NaCl}$ in $\mathrm{D}_{2} \mathrm{O}$. The high ionic strength of $1 \mathrm{M} \mathrm{NaCl}$ was chosen for the ability of the $\mathrm{NaCl}$ ions to screen and reduce double layer repulsion, thereby increasing the propensity of nanoparticles to aggregate, as well as the ability of the mild kosmotropes to reduce hydration and thereby decrease stabilization due to steric and hydration forces. Figure 7 presents the typical SANS spectra for the $3.1 \mathrm{~nm}$ and $4.6 \mathrm{~nm}$ SL-IONP in $1 \mathrm{M} \mathrm{NaCl} \mathrm{D}_{2} \mathrm{O}$ in comparison with SL-IONP in pure $\mathrm{D}_{2} \mathrm{O}$. 


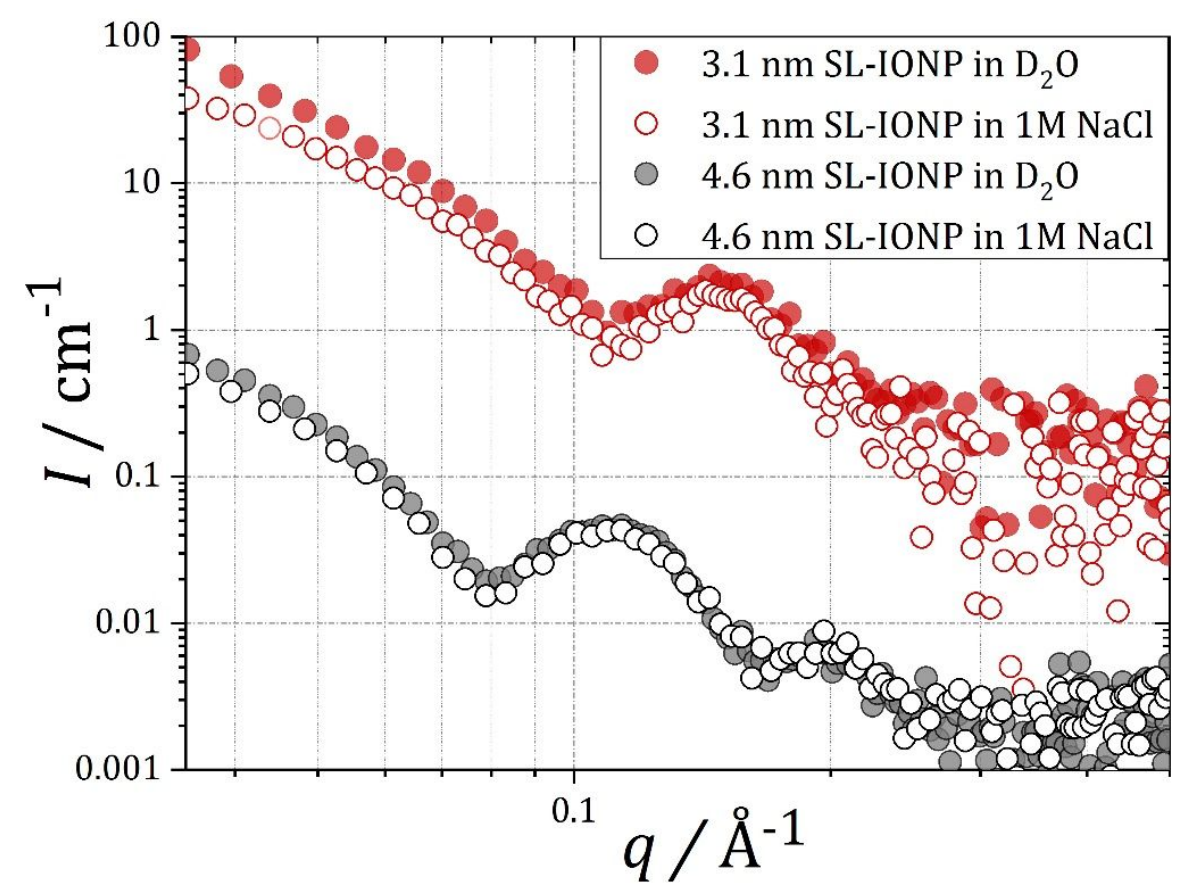

Figure 7. SANS data recorded for $3.1 \mathrm{~nm}$ and $4.6 \mathrm{~nm}$ SL-IONP in $\mathrm{D}_{2} \mathrm{O}$ (red and grey filled circles respectively) and in $1 \mathrm{M} \mathrm{NaCl}$ (dissolved in $\mathrm{D}_{2} \mathrm{O}$ ) (red and grey empty circles respectively).

The data show that the SANS profiles of neither sample change when the ionic strength of the solvent is increased. The absence of change is true in both the mid/high- $q$ region characteristic of the core-shell structure and in the low- $q$ region characteristic of aggregation. This indicates that the SL-NDA shell has neither been removed upon salt addition, nor has its structure been modified. This contrasts to previous findings for 
sophorolipid coated nanoparticles, where aggregation was promoted by the addition of salt. ${ }^{26}$ The improved stability of our novel SL-IONP can be attributed to the NDA anchor chemistry that irreversibly grafts the SL coating densely to the cores. Furthermore, the SANS profiles do not show any sign of particle aggregation, since a structure factor corresponding to particle aggregation would appear in the low $q$-range. There is also no optically visible increase in scattering nor does precipitation take place, which supports the SANS observation of excellent colloidal stability. This is in full agreement with the DLS data that showed retained size and no aggregation and precipitation (cf. Figure 6). The SANS measurements also show that micelles of excess ligand in the $3.1 \mathrm{~nm}$ SL-IONP sample were not altered by that addition of salt (no change in the slope at low $q$ ), which explains why dialysis at high ionic strength could not remove the excess ligand.

In-vitro cellular uptake and cytotoxicity of SL-IONP. To prove the applicability of SLIONP in the biomedical field, we conducted cellular uptake and cytotoxicity tests on two different cell lines: U937 monocytes which are part of the reticuloendothelial system and 
responsible for clearance of pathogens and foreign bodies from the blood and MCF7, a human breast cancer cell line.

Differentiated monocytes, macrophages, are the cells in the body that are most exposed to toxins and foreign bodies. The response of these cells to exposure of SL-IONP is thus of highest relevance. If they detect and scavenge nanoparticles, the nanoparticles will be of limited use in applications that require circulation and/or targeting. It is, to the best of our knowledge, the first time that cellular uptake and toxicity are studied for sophorolipid coated core-shell nanoparticles. Figure 8 shows the results of the cellular uptake and cytotoxicity tests for $4.6 \mathrm{~nm}$ SL-IONP with U937 and MCF7 cells. SL-IONP show very low non-specific uptake by both monocytes and cancer cells. A slight trend to higher uptake of breast cancer cells over the phagocytic monocytes could not be statistically verified. The low uptake is in the same order as previously shown for stealth poly(ethylene glycol)grafted IONP' and can be traced back to the good colloidal stability of the core-shell SLIONP and their resistance against aggregation in presence of proteins and salts (cf. Figure 6 and 7). 
Both sophorolipids and iron oxide nanoparticles have individually shown negligible toxicity at relevant concentrations to various cell lines. ${ }^{57-58}$ Figure $8 \mathrm{~B}$ shows that also SLgrafted $4.6 \mathrm{~nm}$ IONP do not affect the viability of the two tested cell lines. The results suggest an excellent biocompatibility of the SL-IONP.
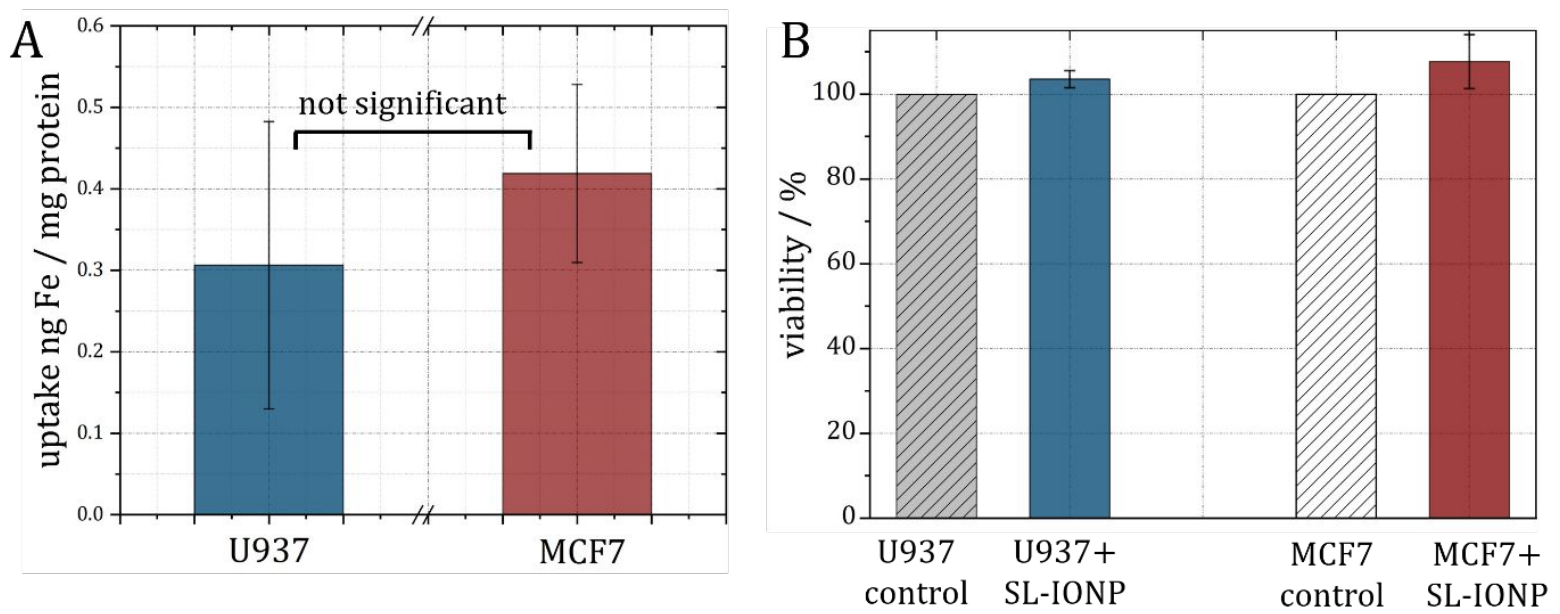

Figure 8. A) Cellular uptake of $4.6 \mathrm{~nm}$ SL-IONP for human monocytes (U937) and human breast cancer cells (MCF7). Negligible uptake was observed with a tendency towards increased uptake in cancer cell lines, but without statistical significance $(p>0.05$ ANOVA 1-way) within the study. B) Cytotoxicity tests for $4.6 \mathrm{~nm}$ SL-IONP with human monocytes (U937) and human breast cancer cells (MCF7). The viability was unchanged for both cell lines. 


\section{Design principles for using SL-NDA to stabilize IONP for applications. In summary, a}

range of challenging conditions for colloidal stability relevant for applications were tested;

all results were consistent with excellent stability of the grafted SL-NDA shell translating

to equally excellent colloidal stability. The low size dispersity, high grafting density and colloidal stability of the SL-IONP system developed here are comparable to those of synthetic glyconanoparticles ${ }^{12,16-17,59-60}$ rather than sophorolipid-capped nanoparticles. ${ }^{23-}$

${ }^{26}$ Our results demonstrate that the densely grafted SL-NDA shell provides a stable repulsive potential preventing both mutual aggregation and protein adsorption under highly challenging conditions $\left(1 \mathrm{M} \mathrm{NaCl}, 70{ }^{\circ} \mathrm{C}\right.$ with and without $\left.\mathrm{FCS}\right)$. The colloidal stability is not adversely affected by temperature and kosmotrope salts that often have a destabilizing effect on polymer coated-core-shell nanoparticles. This is reached by the irreversible grafting of the SL ligand to the core in contrast to pure self-assembly and/or complexation. The expected main contribution to the repulsive interaction of the shell is the high and stable hydration of the lipid head-groups that is apparently strong enough to withstand weakening in the coordination of associated water under relevant challenging 
environmental conditions. This fact is confirmed by the literature on the micellization properties of sugar-based amphiphiles: the strength between the hydrogen bonding between the hydroxyl groups of the sugar unit and water is stronger than between ethylene oxide and water. ${ }^{61}$

It is worth noting that stabilization through a grafted lipid is fundamentally different to the more commonly applied approach of a high- $M w$ flexible polymer; the low thickness added by a lipid monolayer and its insignificant molecular flexibility makes it possible to design such shells much more compact and structurally precise than using solvated polymer. At the same time, it is impressive that equally high colloidal stability, as demonstrated here, can be obtained. The hydrophobic spacer part of the lipid shell is important to reduce core-core van-der-Waals attraction and likely cannot be made too thin without compromising colloidal stability. However, with a hydrophobic interior of the shell, a prerequisite for stability in biofluids is that the lipid shell (the lipid headgroup region) is dense enough to prevent hydrophobic molecules, such as protein denatured by temperature, to adsorb to shell defects exposing the alkyl part of the lipids. We achieved this by the stable NDA-coupling and efficient ligand exchange protocol used to graft the 
SL-NDA shell. As expected, in the absence of aggregates, the SL-IONP showed only weak interaction with cells in-vitro with negligible uptake and no resulting cytotoxicity, thus making these systems promising candidates for the development of IONP-based biomedical applications. These, so-called stealth, nanoparticles can be further functionalized or make use of specific interactions of the exposed saccharide motif to add targeting capability, for which structural and colloidal stability, as well as suppression of non-specific biomolecular interactions, is a prerequisite.

\section{Conclusion}

We have synthesized monodisperse, biocompatible, sophorolipid-grafted core-shell iron oxide nanoparticles with excellent colloidal stability in demanding application environments mimicking the conditions of biological fluids. As-synthesized, oleic acidcoated IONP with precisely tunable sizes and narrow size distributions were successfully grafted with a nitrodopamide-modified sophorolipid ligand by replacement of the oleic acid. Analysis of TGA and cryo-TEM highlighted the challenge of removing excess ligand as sophorolipid forms very stable aggregates of similar size as the grafted nanoparticles 
and with the same physico-chemical characteristics. Cryo-TEM revealed the coexistence of individual, monodisperse, SL-grafted IONP with large and stable SL-NDA aggregates formed by excess ligand that could not be completely removed by excessive purification. However, also close to pure SL-grafted IONP product was obtained for $4.6 \mathrm{~nm}$ IONP cores. The core-shell structure of SL-IONP was analyzed by SAXS and SANS. The resulting data were successfully fitted with a core-shell model. SANS revealed the thickness of the shell, which was found to be in the order of $2.5 \mathrm{~nm}$, in good agreement with a shell composed of a monolayer of SL-NDA grafted to a highly curved surface.

The stable, grafted core-shell architecture presenting the equivalent of a dense sophorolipid membrane at the nanoparticle surface was shown to translate into excellent colloidal stability under demanding conditions such as in dense protein dispersions (serum) at high temperature and at high ionic strength, as shown by DLS and SANS. The resulting colloidally stable and biocompatible SL-IONP are promising materials in the biomedical field through their combination of IONP imaging and therapeutic properties with natural presentation of functional glycosylation, whereas carbohydrate-rich 
nanoparticles can display unique targeting ability towards lectin-rich biological membranes, including tumor cells and pathogenic bacteria.

\title{
ASSOCIATED CONTENT
}

Supporting Information. Additional information on Materials. Control over iron oxide core size by the precursor to surfactant ratio. Detailed synthetic protocols. NMR, IR, TGA, TEM, cryo-TEM and DLS data for SL-NDA and SL-IONP. Photos of colloidal suspensions. Additional SAXS and SANS data with fits.

\author{
AUTHOR INFORMATION
}

Corresponding Authors

*Erik Reimhult: erik.reimhult@boku.ac.at; Niki Baccile: niki.baccile@upmc.fr.

\section{Author Contributions}

$A L, E R$ and NB planned the work. AL and NB performed most of the experiments. AL, ER and NB wrote the manuscript. All authors contributed to and reviewed the manuscript. 
The manuscript was written through contributions of all authors. All authors have given approval to the final version of the manuscript.

\section{Funding Sources}

This work received financial support by the Institut Laue-Langevin (ILL), Grenoble, France, under the experiment number 9-12-481 (D33, D16 beamlines), and by the European Synchrotron Radiation Facility (ESRF), Grenoble, France, under the experiment number SC-4419. The research presented in that paper was funded by the Austrian Science Fund (FWF) project I 3064 and by the European Research Council under the European Union's Seventh Framework Program (FP/2007-2013)/ERC Grant Agreement No. 310034.

\section{ACKNOWLEDGMENT}

This work benefited from the use of the SasView application, originally developed under NSF award DMR-0520547. SasView contains code developed with funding from the European Union's Horizon 2020 research and innovation programme under the SINE2020 project, grant agreement No 654000. We thank the Lawrence Berkeley 
National Laboratory for access to TEM; work at the Molecular Foundry was supported by the Office of Science, Office of Basic Energy Sciences, of the U.S. Department of Energy under Contract No. DE-AC02-05CH11231.We thank Prof. Dieter Baurecht for access to FTIR. We acknowledge the VIBT Extremophile Center for access to TGA.

\author{
ABBREVIATIONS \\ IONP, iron oxide nanoparticles; OA, oleic acid; $\mathrm{Oct}_{2} \mathrm{O}$, dioctylether; $\mathrm{SL}$, sophorolipids; \\ NDA, nitrodopamine/nitrodopamide; SL-NDA, sophorolipid-nitrodopamide; SL-IONP, \\ sophorolipid-nitrodopamide-grafted iron oxide nanoparticles; PEG, poly(ethylene glycol); \\ PBS, Phosphate buffered saline; FCS, fetal calf serum; HR, high-resolution; LR, low- \\ resolution; TEM, transmission electron microscope/microscopy; TGA, thermogravimetric \\ analysis; DLS, dynamic light scattering; NMR, nuclear magnetic resonance; ATR-FTIR, \\ attenuated total reflection - Fourier transform infra-red; SAXS, small angle x-ray \\ scattering; SANS, small angle neutron scattering; SLD, scattering length density; MWCO, \\ molecular weight cut-off; TOC, total organic content
}




\section{REFERENCES}

1. Hong, R. Y.; Feng, B.; Chen, L. L.; Liu, G. H.; Li, H. Z.; Zheng, Y.; Wei, D. G., Synthesis, Characterization and MRI Application of Dextran-Coated Fe3O4 Magnetic Nanoparticles. Biochem. Eng. J. 2008, 42, 290-300.

2. Lacava, L. M.; Lacava, Z. G. M.; Da Silva, M. F.; Silva, O.; Chaves, S. B.; Azevedo, R. B.; Pelegrini, F.; Gansau, C.; Buske, N.; Sabolovic, D.; Morais, P. C., Magnetic Resonance of a Dextran-Coated Magnetic Fluid Intravenously Administered in Mice. Biophys. J. 2001, 80, 2483-2486.

3. Tassa, C.; Shaw, S. Y.; Weissleder, R., Dextran-Coated Iron Oxide Nanoparticles: A Versatile Platform for Targeted Molecular Imaging, Molecular Diagnostics, and Therapy. Acc. Chem. Res. 2011, 44, 842-852.

4. Boni, A.; Bardi, G.; Bertero, A.; Cappello, V.; Emdin, M.; Flori, A.; Gemmi, M.; Innocenti, C.; Menichetti, L.; Sangregorio, C.; Villa, S.; Piazza, V., Design and Optimization of Lipid-Modified Poly(Amidoamine) Dendrimer Coated Iron Oxide Nanoparticles as Probes for Biomedical Applications. Nanoscale 2015, 7, 7307-7317. 5. Amstad, E.; Textor, M.; Reimhult, E., Stabilization and Functionalization of Iron Oxide Nanoparticles for Biomedical Applications. Nanoscale 2011, 3, 2819-2843.

6. Frey, N. A.; Peng, S.; Cheng, K.; Sun, S., Magnetic Nanoparticles: Synthesis, Functionalization, and Applications in Bioimaging and Magnetic Energy Storage. Chem. Soc. Rev. 2009, 38, 2532-2542.

7. Park, J.; Joo, J.; Kwon, S. G.; Jang, Y.; Hyeon, T., Synthesis of Monodisperse Spherical Nanocrystals. Angew. Chem. Int. Ed. 2007, 46, 4630-4660.

8. $\quad$ Lassenberger, A.; Bixner, O.; Gruenewald, T.; Lichtenegger, H.; Zirbs, R.; Reimhult, E., Evaluation of High-Yield Purification Methods on Monodisperse PegGrafted Iron Oxide Nanoparticles. Langmuir 2016, 32, 4259-4269.

9. Lassenberger, A.; Scheberl, A.; Stadlbauer, A.; Stiglbauer, A.; Helbich, T.; Reimhult, E., Individually Stabilized, Superparamagnetic Nanoparticles with Controlled Shell and Size Leading to Exceptional Stealth Properties and High Relaxivities. ACS Appl. Mater. Interfaces 2017, 9, 3343-3353. 
10. Yang, Q.; Lai, S. K., Anti-PEG Immunity: Emergence, Characteristics, and Unaddressed Questions. Wiley interdisciplinary reviews. Nanomed. Nanobiotechnol. 2015, 7, 655-677.

11. Bernardi, A.; Jimenez-Barbero, J.; Casnati, A.; De Castro, C.; Darbre, T.; Fieschi, F.; Finne, J.; Funken, H.; Jaeger, K.-E.; Lahmann, M.; Lindhorst, T. K.; Marradi, M.; Messner, P.; Molinaro, A.; Murphy, P. V.; Nativi, C.; Oscarson, S.; Penades, S.; Peri, F.; Pieters, R. J.; Renaudet, O.; Reymond, J.-L.; Richichi, B.; Rojo, J.; Sansone, F.; Schaffer, C.; Turnbull, W. B.; Velasco-Torrijos, T.; Vidal, S.; Vincent, S.; Wennekes, T.; Zuilhof, H.; Imberty, A., Multivalent Glycoconjugates as Anti-Pathogenic Agents. Chem. Soc. Rev. 2013, 42, 4709-4727.

12. Marradi, M.; Chiodo, F.; Garcia, I.; Penades, S., Glyconanoparticles as Multifunctional and Multimodal Carbohydrate Systems. Chem. Soc. Rev. 2013, 42, 4728-4745.

13. Lee, Y. C.; Lee, R. T., Carbohydrate-Protein Interactions: Basis of Glycobiology. Acc. Chem. Res. 1995, 28, 321-327.

14. Lis, H.; Sharon, N., Lectins: Carbohydrate-Specific Proteins That Mediate Cellular Recognition. Chem. Rev. 1998, 98, 637-674.

15. Cecioni, S.; Imberty, A.; Vidal, S., Glycomimetics Versus Multivalent Glycoconjugates for the Design of High Affinity Lectin Ligands. Chem. Rev. 2015, 115, 525-561.

16. El-Boubbou, K.; Zhu, D. C.; Vasileiou, C.; Borhan, B.; Prosperi, D.; Li, W.; Huang, X., Magnetic Glyco-Nanoparticles: A Tool to Detect, Differentiate, and Unlock the Glyco-Codes of Cancer Via Magnetic Resonance Imaging. J. Am. Chem. Soc. 2010, 132, 4490-4499.

17. Gallo, J.; Garcia, I.; Padro, D.; Arnaiz, B.; Penades, S., Water-Soluble Magnetic Glyconanoparticles Based on Metal-Doped Ferrites Coated with Gold: Synthesis and Characterization. J. Mater. Chem. B 2010, 20, 10010-10020.

18. Dumur, F.; Guerlin, A.; Dumas, E.; Bertin, D.; Gigmes, D.; Mayer, C. R., Controlled Spontaneous Generation of Gold Nanoparticles Assisted by Dual Reducing and Capping Agents. Gold Bulletin 2011, 44, 119-137. 
19. Duan, H.; Wang, D.; Li, Y., Green Chemistry for Nanoparticle Synthesis. Chem. Soc. Rev. 2015, 44, 5778-5792.

20. Manet, S.; Cuvier, A.-S.; Valotteau, C.; Fadda, G. C.; Perez, J.; Karakas, E.; Abel, S.; Baccile, N., Structure of Bolaamphiphile Sophorolipid Micelles Characterized with SAXS, SANS, and MD Simulations. J. Phys. Chem. B 2015, 119, 13113-13133. 21. Chen, J.; Song, X.; Zhang, H.; Qu, Y., Production, Structure Elucidation and Anticancer Properties of Sophorolipid from Wickerhamiella Domercqiae. Enzyme Microb. Technol. 2006, 39, 501-506.

22. Díaz De Rienzo, M. A.; Banat, I. M.; Dolman, B.; Winterburn, J.; Martin, P. J., Sophorolipid Biosurfactants: Possible Uses as Antibacterial and Antibiofilm Agent. New Biotechnol. 2015, 32, 720-726.

23. Kasture, M.; Singh, S.; Patel, P.; Joy, P. A.; Prabhune, A. A.; Ramana, C. V.; Prasad, B. L. V., Multiutility Sophorolipids as Nanoparticle Capping Agents: Synthesis of Stable and Water Dispersible Co Nanoparticles. Langmuir 2007, 23, 11409-11412. 24. Singh, S.; Patel, P.; Jaiswal, S.; Prabhune, A. A.; Ramana, C. V.; Prasad, B. L. V., A Direct Method for the Preparation of Glycolipid-Metal Nanoparticle Conjugates: Sophorolipids as Reducing and Capping Agents for the Synthesis of Water ReDispersible Silver Nanoparticles and Their Antibacterial Activity. New J. Chem. 2009, 33, 646-652.

25. Kumar, D. V. R.; Kasture, M.; Prabhune, A. A.; Ramana, C. V.; Prasad, B. L. V.; Kulkarni, A. A., Continuous Flow Synthesis of Functionalized Silver Nanoparticles Using Bifunctional Biosurfactants. Green Chem. 2010, 12, 609-615.

26. Baccile, N.; Noiville, R.; Stievano, L.; Bogaert, I. V., Sophorolipids-Functionalized Iron Oxide Nanoparticles. Phys. Chem. Chem. Phys. 2013, 15, 1606-1620.

27. Amstad, E.; Gehring, A. U.; Fischer, H.; Nagaiyanallur, V. V.; Hahner, G.; Textor, M.; Reimhult, E., Influence of Electronegative Substituents on the Binding Affinity of Catechol-Derived Anchors to Fe3O4 Nanoparticles. J. Phys. Chem. C 2011, 115, 683691.

28. Amstad, E.; Gillich, T.; Bilecka, I.; Textor, M.; Reimhult, E., Ultrastable Iron Oxide Nanoparticle Colloidal Suspensions Using Dispersants with Catechol-Derived Anchor Groups. Nano Letters 2009, 9, 4042-4048. 
29. Baccile, N.; Cuvier, A.-S.; Valotteau, C.; Van Bogaert, I. N. A., Practical Methods to Reduce Impurities for Gram-Scale Amounts of Acidic Sophorolipid Biosurfactants. Eur. J. Lipid Sci. Technol. 2013, 115, 1404-1412.

30. Dhasaiyan, P.; Le Griel, P.; Roelants, S.; Redant, E.; Van Bogaert, I. N. A.; Prevost, S.; Prasad, B. L. V.; Baccile, N., Micelles Versus Ribbons: How Congeners Drive the Self-Assembly of Acidic Sophorolipid Biosurfactants. Chem. Phys. Chem. 2017, 18, 643-652.

31. Napolitano, A.; D'Ischia, M.; Costantini, C.; Prota, G., A New Oxidation Pathway of the Neurotoxin 6-Aminodopamine. Isolation and Characterization of a Dimer with a Tetrahydro[3,4a]lminoethanophenoxazine Ring System. Tetrahedron 1992, 48, 85158522.

32. Bixner, O.; Lassenberger, A.; Baurecht, D.; Reimhult, E., Complete Exchange of the Hydrophobic Dispersant Shell on Monodisperse Superparamagnetic Iron Oxide Nanoparticles. Langmuir 2015, 31, 9198-9204.

33. El-Faham, A.; Funosas, R. S.; Prohens, R.; Albericio, F., Comu: A Safer and More Effective Replacement for Benzotriazole-Based Uronium Coupling Reagents. Chem. - Eur. J. 2009, 15, 9404-9416.

34. Kurzhals, S.; Zirbs, R.; Reimhult, E., Synthesis and Magneto-Thermal Actuation of Iron Oxide Core-Pnipam Shell Nanoparticles. ACS App. Mater. Interfaces 2015, 7, $19342-19352$.

35. Hyeon, T.; Lee, S. S.; Park, J.; Chung, Y.; Na, H. B., Synthesis of Highly Crystalline and Monodisperse Maghemite Nanocrystallites without a Size-Selection Process. J. Am. Chem. Soc. 2001, 123, 12798-12801.

36. Park, J.; An, K.; Hwang, Y.; Park, J.-G.; Noh, H.-J.; Kim, J.-Y.; Park, J.-H.; Hwang, N.-M.; Hyeon, T., Ultra-Large-Scale Syntheses of Monodisperse Nanocrystals. Nat. Mater. 2004, 3, 891-895.

37. Mondini, S.; Ferretti, A. M.; Puglisi, A.; Ponti, A., Pebbles and Pebblejuggler: Software for Accurate, Unbiased, and Fast Measurement and Analysis of Nanoparticle Morphology from Transmission Electron Microscopy (TEM) Micrographs. Nanoscale 2012, 4, 5356-5372. 
38. Guinier, A.; Fournet, G., Small-Angle Scattering of X-Rays. John Wiley and Sons: New York, 1955.

39. http://www.sasview.org.

40. Finger, L. W.; Hazen, R. M.; Hofmeister, A. M., High-Pressure Crystal Chemistry of Spinel (MgAl2O4) and Magnetite (Fe3O4): Comparisons with Silicate Spinels. Phys. Chem. Miner. 1986, 13, 215-220.

41. Gulley-Stahl, H.; Hogan, P. A., 2nd; Schmidt, W. L.; Wall, S. J.; Buhrlage, A.; Bullen, H. A., Surface Complexation of Catechol to Metal Oxides: An ATR-FTIR, Adsorption, and Dissolution Study. Environ. Sci. Technol. 2010, 44, 4116-4121.

42. Valotteau, C.; Calers, C.; Casale, S.; Berton, J.; Stevens, C. V.; Babonneau, F.; Pradier, C.-M.; Humblot, V.; Baccile, N., Biocidal Properties of a Glycosylated Surface: Sophorolipids on Au(111). ACS Appl. Mater. Interfaces 2015, 7, 18086-18095.

43. Slovetskii, V. I., Ir Spectra of Nitro Compounds. Bull. Acad. Sci. USSR, Div. Chem. Sci. 1970, 19, 2086-2091.

44. Shirmardi Shaghasemi, B.; Dehghani, E. S.; Benetti, E. M.; Reimhult, E., HostGuest Driven Ligand Replacement on Monodisperse Inorganic Nanoparticles. Nanoscale 2017, 9, 8925-8929.

45. Santos-Carballal, D.; Roldan, A.; Grau-Crespo, R.; de Leeuw, N. H., A Dft Study of the Structures, Stabilities and Redox Behaviour of the Major Surfaces of Magnetite Fe3O4. Phys. Chem. Chem. Phys. 2014, 16, 21082-21097.

46. Valotteau, C.; Banat, I. M.; Mitchell, C. A.; Lydon, H.; Marchant, R.; Babonneau, F.; Pradier, C.-M.; Baccile, N.; Humblot, V., Antibacterial Properties of SophorolipidModified Gold Surfaces against Gram Positive and Gram Negative Pathogens. Colloids Surf. B 2017, 157, 325-334.

47. Chen, M.; Dong, C.; Penfold, J.; Thomas, R. K.; Smyth, T. J. P.; Perfumo, A.; Marchant, R.; Banat, I. M.; Stevenson, P.; Parry, A.; Tucker, I.; Campbell, R. A., Adsorption of Sophorolipid Biosurfactants on Their Own and Mixed with Sodium Dodecyl Benzene Sulfonate, at the Air/Water Interface. Langmuir 2011, 27, 8854-8866. 48. Baccile, N.; Selmane, M.; Le Griel, P.; Prevost, S.; Perez, J.; Stevens, C. V.; Delbeke, E.; Zibek, S.; Guenther, M.; Soetaert, W.; Van Bogaert, I. N. A.; Roelants, S., 
Ph-Driven Self-Assembly of Acidic Microbial Glycolipids. Langmuir 2016, 32, 63436359.

49. Abel, S.; Dupradeau, F.-Y.; Raman, E. P.; MacKerell, A. D.; Marchi, M., Molecular Simulations of Dodecyl-B-Maltoside Micelles in Water: Influence of the Headgroup Conformation and Force Field Parameters. J. Phys. Chem. B 2011, 115, 487-499.

50. Bergin, R.; Carlstrom, D., Structure of the Pyrocatecholamines. Ii. Crystal Structure of Dopamine Hydrochloride. Acta Crystallogr., Sect. B 1968, 24, 1506-1510. 51. Smith, M. B.; McGillivray, D. J.; Genzer, J.; Loesche, M.; Kilpatrick, P. K., Neutron Reflectometry of Supported Hybrid Bilayers with Inserted Peptide. Soft Matter 2010, 6, 862-865.

52. Reimhult, E.; Schroffenegger, M.; Lassenberger, A., Design Principles for Thermoresponsive Core-Shell Nanoparticles - Controlling Thermal Transitions by Brush Morphology. Langmuir 2019, 22, 7092-7104

53. Manet, S.; Cuvier, A.-S.; Valotteau, C.; Fadda, G. C.; Perez, J.; Karakas, E.; Abel, S.; Baccile, N., Structure of Bolaamphiphile Sophorolipid Micelles Characterized with SAXS, SANS, and MD Simulations. J. Phys. Chem. B 2015, 119, 13113-13133.

54. Penfold, J.; Chen, M.; Thomas, R. K.; Dong, C.; Smyth, T. J.; Perfumo, A.; Marchant, R.; Banat, I. M.; Stevenson, P.; Parry, A.; Tucker, I.; Grillo, I., Solution SelfAssembly of the Sophorolipid Biosurfactant and Its Mixture with Anionic Surfactant Sodium Dodecyl Benzene Sulfonate. Langmuir 2011, 27, 8867-8877.

55. Amstad, E.; Gillich, T.; Bilecka, I.; Textor, M.; Reimhult, E., Ultrastable Iron Oxide Nanoparticle Colloidal Suspensions Using Dispersants with Catechol-Derived Anchor Groups. Nano Letters 2009, 9, 4042-4048.

56. Zirbs, R.; Lassenberger, A.; Vonderhaid, I.; Kurzhals, S.; Reimhult, E., MeltGrafting for the Synthesis of Core-Shell Nanoparticles with Ultra-High Dispersant Density. Nanoscale 2015, 7, 11216-11225.

57. Ikeda, Y.; Sunakawa, T.; Tsuchiya, S.; Kondo, M.; Okamoto, K., Toxicological Studies on Sophorolipid Derivatives. (I). Acute Toxicity, Eye Irritation, Primary Skin Irritation, Skin Sensitization, Phototoxicity, Photosensitization, Mutagenicity of 
Polyoxypropylene (12) [(2'-0-Beta-D-Glucopyranosyl-Beta-D-Glucopyranosyl)Oxy-] Fatty Acid Ester-]. J. toxicol. Sci. 1986, 11, 197-211.

58. Gal, N.; Lassenberger, A.; Herrero-Nogareda, L.; Scheberl, A.; Charwat, V.; Kasper, C.; Reimhult, E., Interaction of Size-Tailored PEGylated Iron Oxide Nanoparticles with Lipid Membranes and Cells. ACS Biomat. Sci. Eng. 2017, 3, 249259.

59. Varela-Aramburu, S.; Wirth, R.; Lai, C. H.; Orts-Gil, G.; Seeberger, P. H., Straightforward and Robust Synthesis of Monodisperse Surface-Functionalized Gold Nanoclusters. Beilstein J. Nanotech. 2016, 7, 1278-1283.

60. Reynolds, M.; Marradi, M.; Imberty, A.; Penades, S.; Perez, S., Influence of Ligand Presentation Density on the Molecular Recognition of Mannose-Functionalised Glyconanoparticles by Bacterial Lectin Bc2l-A. Glycoconj. J. 2013, 30, 747-757.

61. Ruiz, C. C., Sugar-Based Surfactants; Fundamentals and Applications. [In: Surfactant Sci. Ser., 2009; 143]. CRC Press: 2008.

\section{Graphical Abstract}

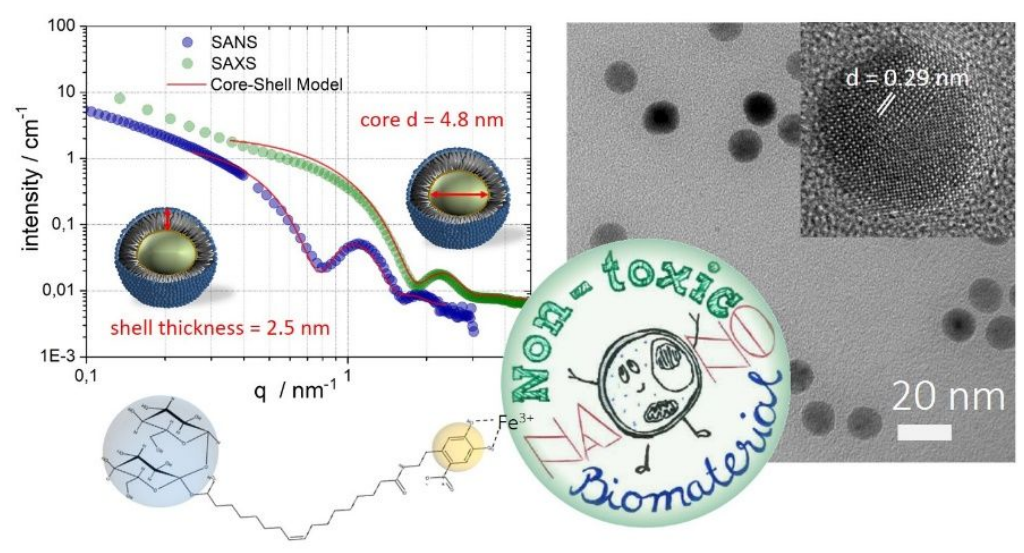

